\title{
Aeroacoustic Simulations of a Nose Landing Gear with FUN3D: A Grid Refinement Study
}

\author{
Veer N. Vatsa* \\ Mehdi R. Khorrami* \\ and \\ David P. Lockard ${ }^{\dagger}$ \\ NASA Langley Research Center, Hampton, VA 23681
}

\begin{abstract}
A systematic grid refinement study is presented for numerical simulations of a partially-dressed, cavity-closed (PDCC) nose landing gear configuration that was tested in the University of Florida's open-jet acoustic facility known as the UFAFF. The unstructured-grid flow solver FUN3D is used to compute the unsteady flow field for this configuration. Mixed-element grids generated using the Pointwise ${ }^{\circledR}$ grid generation software are used for numerical simulations. Particular care is taken to ensure quality cells and proper resolution in critical areas of interest in an effort to minimize errors introduced by numerical artifacts. A set of grids was generated in this manner to create a family of uniformly refined grids. The finest grid was then modified to coarsen the wall-normal spacing to create a grid suitable for the wall-function implementation in FUN3D code. A hybrid Reynolds-averaged Navier-Stokes/large eddy simulation (RANS/LES) turbulence modeling approach is used for these simulations. Time-averaged and instantaneous solutions obtained on these grids are compared with the measured data. These CFD solutions are used as input to a Ffowes Williams-Hawkings (FW-H) noise propagation code to compute the farfield noise levels. The agreement of the computed results with the experimental data improves as the grid is refined.
\end{abstract}

\section{Nomenclature}

BART Basic Aerodynamic Research Tunnel

BDF2 second-order backward differencing formulation

BDF2OPT optimized second-order backward differencing formulation

CFD computational fluid dynamics

$C_{p} \quad$ Coefficient of pressure, $\left[\left(p-p_{\infty}\right) /\left(0.5 \rho_{\infty} u_{\infty}{ }^{2}\right)\right]$

DES detached eddy simulation

DDES delayed detached eddy simulation

FW-H Ffowcs Williams-Hawkings

LES large eddy simulation

MDDES modified delayed detached eddy simulation

PDCC partially-dressed, cavity-closed

PIV particle image velocimetry

PSD power spectral density

RANS Reynolds-averaged Navier-Stokes

SPL sound pressure level

TKE turbulence kinetic energy, $\left[\left(u^{\prime 2}+v^{\prime 2}+w^{\prime 2}\right) / 2\right]$

UFAFF University of Florida Aeroacoustic Flow Facility

$u, v, w \quad$ Cartesian fluid velocity components

$X, Y, Z \quad$ Cartesian coordinates

2-D TKE two-dimensional turbulence kinetic energy, $\left[\left(u^{\prime 2}+v^{\prime 2}\right) / 2\right]$

$\theta \quad$ circumferential angle, measured clockwise from wheel leading edge

$\rho \quad$ density

\footnotetext{
*Aerospace Engineer, Computational AeroSciences Branch, Research Directorate; Associate Fellow AIAA
}

${ }^{\dagger}$ Aerospace Engineer, Computational AeroSciences Branch, Research Directorate; Senior Member AIAA 


\section{Superscript:}

perturbation quantity (e.g., $u^{\prime}=u-u_{\infty}$ )

\section{Subscript:}

$\infty$

freestream quantity

\section{Introduction}

In recent years, airframe noise has become a significant, if not the primary source of commercial aircraft noise during the approach to landing. Hence, there has been a growing emphasis on reducing airframe noise to meet the increasingly aggressive standards for permissible noise levels near airports. A significant contributor to airframe noise during approach and landing for commercial aircraft ${ }^{1}$ is the landing gear; therefore, understanding noise sources associated with such configurations is very important. Prediction of noise sources around the landing gear of an aircraft is an extremely challenging problem despite the progress made during recent years in the field of computational fluid dynamics (CFD). The geometric complexity and highly chaotic unsteady flow fields associated with landing gear pose significant challenges for numerical simulation.

Despite these difficulties, multiple groups have undertaken the task of simulating flows over such complex landing gear configurations with varying degrees of success in predicting the associated flow field and the farfield noise. ${ }^{2-7}$ A literature survey indicates that the quality of numerical solutions for such configurations has improved steadily over the span of the last few years, as the researchers have performed computations on increasingly finer grids. However, systematic grid refinement studies for these types of configurations are almost nonexistent due to the geometric complexity and scale variation of the associated components.

For the current work, we make use of the unstructured-grid flow code FUN3D ${ }^{8}$ in which the compressible NavierStokes equations are solved to simulate the viscous unsteady flow field. The FUN3D code has been used for many large-scale applications. ${ }^{9}$ These include simulations of unsteady flow past single- and tandem-cylinder configurations, ${ }^{10}$ where time-averaged and perturbation quantities were shown to be in good agreement with experimental data and earlier computations from the well-established, structured-grid flow code CFL3D. ${ }^{11}$ The FUN3D code has also been used to solve the flow over nose landing gear configurations, and the resulting solutions were shown to compare well with experimental data. ${ }^{6,7}$ The grids for the nose landing gear configuration in these studies were generated with the widely used VGRID code developed by Pirzadeh, ${ }^{12}$ but the mesh generation required significant human effort and time. To reduce the total time to solution, an attempt was made by Vatsa et al. ${ }^{13}$ to examine the effect of off-body grid refinement for a Gulfstream aircraft nose landing gear configuration with the feature-based off-body grid adaption technique available in FUN3D. It was demonstrated through this work that comparable levels of accuracy can be obtained more efficiently on smaller sized grids by using automatic grid adaption in high gradient regions. However, based on this study, it became clear that further improvement in accuracy for a given numerical scheme would require surface grid refinement in addition to off-body grid adaption.

An alternative grid generation technique was investigated in a recent paper ${ }^{14}$ where we demonstrated the application of the FUN3D solver for this landing gear case on a 62 million node mixed-element grid generated by the Pointwise ${ }^{\circledR}$ grid generation code. Judging by the agreement with the experimental data, these solutions were shown to have a level of accuracy comparable to previously available solutions on much finer grids (in terms of number of grid points) obtained from different grid generation techniques. By changing just a few parameters in the Pointwise ${ }^{\circledR}$ scripts, one can refine or coarsen the grids uniformly in all three coordinate directions to produce a family of grids suitable for a systematic grid refinement study. The results on such a family of uniformly refined grids are presented here for the Gulfstream aircraft nose landing gear configuration that has been tested in multiple wind tunnels, and has been the subject of numerous numerical studies cited earlier in this paper.

\section{Governing Equations and Flow Solver}

In the present work, the spatially second-order accurate CFD solver FUN3D is used to obtain numerical solutions of the unsteady Navier-Stokes equations. A hybrid approach is used where the Reynolds-averaged Navier-Stokes (RANS) equations supplemented by turbulence models are solved in regions where the grid is inadequate to resolve the unsteady flow features, such as near solid walls, and a large eddy simulation (LES) approach is used in the computational domain away from the wall boundary layer regions. The details of the blending between these regions and the subgrid scale model are dependent on the turbulence model used for simulations. ${ }^{15,16}$ 
The unstructured-grid flow solver was developed originally by Anderson and Bonhaus, ${ }^{17}$ and has gone through significant modifications over the years by a team of NASA Langley Research Center researchers. ${ }^{18}$ For the present simulations, the discrete form of the governing equations is solved in a time-accurate manner with a constant time step at every grid point. At each iteration step, a linear system of equations is relaxed with a point implicit procedure. ${ }^{19}$ Roe's flux-difference splitting scheme ${ }^{20}$ is used here without a flux limiter.

A dual time-stepping algorithm ${ }^{21}$ with subiterations is employed in FUN3D to converge the solution within each time step. For these simulations, 15 subiterations per time step were used, which was adequate to obtain at least three orders of magnitude reduction in the residuals of the governing equations. The time step was chosen to adequately resolve the unsteady signals up to $10 \mathrm{kHz}$ in frequency. An optimized second-order backward differencing formulation (BDF2OPT) scheme $^{22}$ was chosen for advancing the solution in time. This scheme produces higher temporal accuracy compared to the standard second-order backward differencing (BDF2) scheme at nominally the same computational cost but with slightly increased memory usage ( $<1 \%$ of total memory) required for storing a set of primary flow and turbulence variables at an extra time instance.

The turbulence model under consideration here is based on the one-equation Detached Eddy Simulation (DES) model of Spalart. ${ }^{23}$ The DES model has been subsequently modified by Spalart et al. ${ }^{24}$ to overcome some of the shortcomings related to its grid dependence resulting in a new version of the model named Delayed Detached Eddy Simulation or DDES. In the DDES model, a blending function that varies between 0 for RANS mode and 1 for LES mode is applied to the destruction terms of the turbulence model. The equation for the turbulent variable $\tilde{\nu}$ for the DDES formulation of Spalart-Allmaras model is written in the dimensional form as:

$$
\frac{\partial \tilde{\nu}}{\partial t}+u_{j} \frac{\partial \tilde{\nu}}{\partial x_{j}}=c_{b 1}\left(1.0-f_{t 2}\right) \tilde{S} \tilde{\nu}-\left[c_{w 1} f_{w}-\frac{c_{b 1}}{\kappa^{2}} f_{t 2}\right]\left(\frac{\tilde{\nu}}{\tilde{d}}\right)^{2}+\frac{1}{\sigma}\left[\frac{\partial}{\partial x_{j}}\left((\nu+\tilde{\nu}) \frac{\partial \tilde{\nu}}{\partial x_{j}}\right)+c_{b 2} \frac{\partial \tilde{\nu}}{\partial x_{i}} \frac{\partial \tilde{\nu}}{\partial x_{i}}\right]+f_{t 1} \Delta U^{2}
$$

and the turbulent eddy viscosity can be computed from the relation

$$
\mu_{t}=\rho \tilde{\nu} f_{v 1}
$$

where

$$
\begin{gathered}
\chi=\frac{\tilde{\nu}}{\nu}, \quad \tilde{S}=\Omega+\hat{s}, \hat{s}=\frac{\tilde{\nu}}{\kappa^{2} \tilde{d}^{2}} f_{v 2}, \quad f_{v 1}=\frac{\chi^{3}}{\chi^{3}+c_{v 1}^{3}}, \quad f_{v 2}=1-\frac{\chi}{1+\chi f_{v 1}}, f_{w}=g\left[\frac{1+c_{w 3}^{6}}{g^{6}+c_{w 3}^{6}}\right]^{1 / 6}, \\
g=r+c_{w 2}\left(r^{6}-r\right), \quad r=\min \left[\frac{\tilde{\nu}}{\tilde{S} \kappa^{2} \tilde{d}^{2}}, 10\right], \quad f_{t 2}=c_{t 3} \exp \left(-c_{t 4} \chi^{2}\right), \quad r_{d}=\frac{\nu_{t}+\nu}{\sqrt{U_{i, j} U_{i, j}} \kappa^{2} d^{2}}, \\
f_{d}=1-\tanh \left(\left[8 r_{d}\right]^{3}\right), \tilde{d}=d-f_{d} \max \left(0, d-C_{D E S} \Delta\right), \Delta=\max (\Delta x, \Delta y, \Delta z)
\end{gathered}
$$

Here, $\rho$ is the density, $\nu=\mu / \rho$ is the molecular kinematic viscosity, $\mu$ is the molecular dynamic viscosity, $\Omega$ is the magnitude of the vorticity, $U_{i, j}=\frac{\partial U_{i}}{\partial x_{j}}$ is the (i,j)'th component of stress tensor, and $d$ is the distance from the field point to the nearest wall. The term, $f_{d}$, is known as the shielding function, and is designed to remain close to zero in the inner part of the boundary layer (RANS) region and then slowly rise to a value of 1 in the outer portion of viscous layer. The value of various coefficients used in the turbulence model are readily available in Ref. 24.

The last term in Eq. (1) is referred to as the trip term, and most CFD codes, including the FUN3D code, ignore this term due to practical difficulties, since it requires apriori knowledge of transition location. A slight modification of the S-A DDES model was suggested by Vatsa and Lockard ${ }^{10}$ to overcome numerical difficulties associated with the nonphysical behavior of the eddy viscosity observed in the upstream region of cylindrical bluff bodies. In this modified model, the blending function suggested by Spalart et al. ${ }^{24}$ for the DDES model for the destruction terms is also applied to the production terms of the turbulence model, and it will be referred to as the modified DDES, or "MDDES" model, in this paper.

\section{Simulated Configuration and Grid Generation Methodology}

The configuration under consideration here is the 1/4-scale, high-fidelity replica of a partially-dressed closed-cavity (PDCC) Gulfstream aircraft nose landing gear that was tested by Zawodny et al. ${ }^{25}$ in the University of Florida's Aeroacoustic Flow Facility (UFAFF), an anechoic open-jet tunnel. Particle Image Velocimetry (PIV) measurements for the same model were made in the Basic Aerodynamic Research Tunnel (BART) tunnel ${ }^{26}$ at NASA Langley Research Center. A schematic of the computational domain chosen for current simulations of the nose landing gear configuration in the UFAFF is shown in Fig. 1. In these simulations, the nose gear assembly is attached to a flat mounting plate, which is 
suspended in space. The computational domain enclosing the gear assembly and mounting plate is comprised of inflow, outflow, top, bottom and side boundaries as shown in Fig. 1.

The baseline grid for this work was created using Pointwise ${ }^{\circledR}$ version 17.2R 2 and contained 62 million nodes. This mixed-element grid consisted of mostly prismatic cells near solid surfaces, which were connected by pyramid shaped cells to tetrahedrons in regions away from the boundary layers. The nominal value of $y^{+}$for this grid is approximately 1 , and is well suited for standard viscous wall treatment, where the governing equations are integrated to the wall. Due to the dynamic nature of the processes responsible for noise generation, fairly stringent constraints were placed on the computational mesh beyond those typically associated with attached turbulent flows. Most notably, regions of flow separation must maintain a high density of points away from the body to reduce numerical diffusion. This is of particular importance in areas where the separated wake impinges on material surfaces downstream. Targeting critical regions of interest and locally refining the grid provides the benefits of off-body resolution for improved numerical accuracy without an undue increase in overall node count. As discussed by Vatsa et al., ${ }^{14}$ baffle surfaces were used in critical off-body regions, such as wheel cavity regions, to control local grid size in unstructured blocks.

An attempt was made to create a family of grids, one coarser and one finer grid compared to the baseline 62 million node grid by simply changing the spacing parameters and dimensions used in the Pointwise ${ }^{\circledR}$ script. The idea was to refine (or coarsen) the grid uniformly in all three coordinate directions including the surface grid. It resulted in a coarse grid of 24.5 million nodes and a fine grid consisting of 142 million nodes, thus resulting in approximately a factor of 5.8 variation in the overall grid size. The nominal value of $y^{+}$for this family of grids is in the range of $0.75-1.25$. An additional grid replicating the surface grid density of the 142 million node grid was created for calibrating the accuracy of the wall function approach in the FUN3D code by coarsening the grid spacing normal to solid surfaces. The nominal value of $y^{+}$for this grid is 25 , which resulted in a grid count of 104 million nodes.

Planar cuts at the torque arm and wheel midplane (at locations marked schematically in Fig. 2) are shown in Figs. 3-4 to display the variation in the coarsest to finest grids used here. Exploded views of these grids in a small, focus region aft of the wheel midplane (marked on Fig. 4) are also presented in Fig. 5 for detailed comparisons of the grid distributions in the vicinity of the wheels. In addition, to better illustrate the grid density variation on solid surfaces as the grid count is increased, grid distributions on the top portion of the starboard wheel are presented in Fig. 6. One can clearly see a systematic increase in resolution while going from 24 to 142 million nodes. The differences in resolution between the 142 million node grid $\left(y^{+}=0.75\right)$ and the 104 million node grid $\left(y^{+}=25\right)$ grid are apparent only in narrow off-surface regions, such as the ones shown in Fig. 5.

\section{Results}

To match the experimental test conditions, the computations were performed at a freestream Mach number of 0.166 and a Reynolds number of $7.3 \times 10^{4}$ based on the main strut (piston) diameter of 0.75 inches. The computational domain chosen for numerical solutions (see Fig. 1) represents the open jet (no tunnel wall solid boundaries) test set up of the UFAFF tunnel. The computations were run in a fully-turbulent mode to simulate the flow conditions commensurate with the use of transition strip on the shock strut, and the relatively rough surfaces of the tested model. A constant wall temperature based on flat plate adiabatic wall conditions and no-slip conditions were imposed on viscous surfaces, which included the gear, fuselage and mounting plate. At the inflow plane, total pressure and total temperature corresponding to the wind tunnel conditions were imposed. ${ }^{27}$ Farfield conditions based on Riemann invariants were imposed at the outflow, sides, top, and bottom boundaries.

The FUN3D flow solver is run first in a steady mode starting from freestream conditions to expedite the development of the mean flow. The solver is then run in time-accurate (unsteady) mode for a sufficient number of time steps to purge the initial disturbances from the computational domain before performing time-averaging of flow quantities. The unsteady data at solid surfaces is collected at every other time step for approximately 0.3 seconds of physical time to perform aeroacoustic analysis. All of the computations were run with a time step of approximately $5 \times 10^{-6}$ seconds so as to resolve unsteady signals up to a frequency of $10 \mathrm{kHz}$.

During the course of this study, unexpected behavior was observed in the 142 million node grid solutions. It appears that as the grid is refined, the transition from laminar to turbulent flow moves downstream of the landing gear wheels past the top dead center of the wheel, which produces nonphysical results. This delay in transition occurs despite the fact that the flow code is being run in a fully turbulent mode with an inflow value of $\tilde{\nu}$ being specified as 3 times the freestream molecular viscosity. Apparently, the destruction term causes a decay of $\tilde{\nu}$ in the approach region on finer grids during Detached-Eddy Simulations, and can bring the value of $\chi$ into the basin of attraction of the value 0, i.e., $1-f_{t 2}<0$. This is more likely to occur at moderate Reynolds numbers on very fine grids. When this situation occurs, a simulation intended to be fully turbulent can produce a laminar boundary layer contrary to the user's intentions. 
A separate study was conducted by Vatsa, Lockard and Spalart ${ }^{28}$ to examine the grid sensitivity of the SA-based DDES turbulence model for flow over simple blunt bodies. Based on the solutions over a circular cylinder and an isolated landing gear wheel used in Ref. 28, it became clear that as the grid is refined, the shielding function $\left(f_{d}\right)$ in Eq. (1) remains near 1 across the entire boundary layer height, although it was designed to remain near zero in the inner part of the boundary layer region (RANS) and then slowly rise to a value of 1 in the outer portion of viscous layer. This behavior is contrary to the original intent of the DDES model, and produces nonphysical results on finer grids, where the eddy viscosity in the boundary layer stays near zero beyond the top dead center of blunt bodies, before eventual transition to turbulent flow due to adverse pressure gradient. As suggested in Ref. 28, the simplest solution to avoid this situation and to reduce the effect of grid refinement on the transition location is to drop the $f_{t 2}$ term from the turbulence model. It is worth noting that the $f_{t 2}$ term is only needed when the $f_{t 1}$ trip term is included in the SA model, and simulations are run with very low values of freestream turbulence levels. Since the $f_{t 1}$ trip term requires specification of transition location, which is generally not known for complex configurations, FUN3D and many other CFD codes do not include this term. The SA model without the $f_{t 2}$ term is known as the SA-noft2 version, as per the nomenclature of the Turbulence Modeling Resource, (TMR). ${ }^{29}$ All of the solutions presented in this paper were obtained with the SA-noft2-based MDDES model and a value of $\tilde{\nu}$ being specified as 3 times the freestream molecular viscosity at the inflow plane to simulate fully turbulent flows.

As a first set of quantitative comparisons, the computed time-averaged surface pressure distributions on the port wheel are compared with experimental data in Fig. 7. The $C_{p}$ distributions in this figure are plotted as a function of the circumferential angle $\theta$, which is measured in the clockwise direction from the wheel leading edge. Computed results for the different grids are compared here with the experimental data. In general, the agreement with experimental data is shown to improve with grid refinement, with the biggest improvement occurring when increasing the grid density from 24 million to 62 million nodes. Only minor differences are observed between the solutions on the 62 and 142 million node grids. Finally, the $C_{p}$ distributions obtained on the 104 million node wall function grid $\left(y^{+}=25\right)$ are found to be almost indistinguishable from the 142 million node grid solution except in the vicinity of $\theta=230^{\circ}$.

Instantaneous streamwise velocity contours for the torque arm planar cuts of Figs. 3 (a)-(d) are presented in Figs. 8 (a)(d). Although these snapshots can not be used for direct comparisons due to different time evolution histories, they are still useful for qualitative comparisons. The resolution of wakes and shear layers created by the upstream strut and the torque arm indicates significant improvement between the 24 million and 62 million node grids. As expected, the finest structures for wakes and shear layers are observed in the 142 million node grid solutions. The resolution of wake and shear layers in the 104 million node wall function solutions appear to be comparable to the 142 million node solutions. The far-wake region for these grids are not as well resolved as the near-wake region, but being far downstream of the gear assembly, coarser resolution in that region is not expected to have a significant effect on perturbation pressures on the landing gear and the farfield noise.

The time-averaged streamwise velocity contours along the torque arm cut are compared for the 24 and 142 million node grids in Fig. 9. Results for the 104 million node wall function grid are also included in this figure. In addition, results from PIV measurements acquired in the BART tunnel ${ }^{26}$ for this configuration are also presented in this figure for comparison. The contour plots for the 142 million node case capture some of the finer details in the wake region that are missing in the 24 million node results. On the other hand, results on the 104 million node grid are almost indistinguishable from the 142 million grid case. Although the computed results indicate somewhat larger low velocity regions in the near wake compared to the PIV data, the overall shape and growth of the shear layer in the wake region is well predicted. Some of the subtle differences, especially underprediction of the velocities in the farfield, can be attributed to the fact that these computations are for free air, whereas the PIV data was acquired in a closed wall tunnel in which downstream flow accelerates due to tunnel blockage.

Similar comparisons for the time-averaged velocities are presented along a plane downstream of the midsection of the wheels in Fig. 10. Once again, the overall agreement of computed results with the measured velocity data is quite good. The edge of the wake for the 24 million node case shows steeper rate of decay and an additional pocket of very low velocities in the near wake compared to the 142 million grid results. On the other hand, differences between the 142 and 104 million grid results are minimal.

The two-dimensional turbulence kinetic energy (2-D TKE), $\left[\left(u_{r m s}^{\prime}\right)^{2}+\left(v_{r m s}^{\prime}\right)^{2}\right] / 2$, contours on these grids along the same planar wheel-wake cut are presented in Fig. 11. These 2-D TKE distributions indicate high levels in the shear layers developing at the edges of wheels, which then decays further downstream. High concentration of 2-D TKE is also observed in the gap between the wheels, which most probably is caused by the high level of unsteady fluctuations in the wake region downstream of the strut and wheel axle. The largest difference in the 24 and 142 million grid solutions is observed in the region just downstream of the gap between the wheels. Other than the subtle differences in the midwake region, agreement between the results for the 142 and 104 million grid cases are quite small. The overall agreement of the numerical solutions with the experimental data, including the maximum level and streamwise decay rate of 2-D TKE 
is quite good.

Next, we examine the surface pressure power spectral density (PSD) distributions at different locations on the gear corresponding to the position of Kulite ${ }^{\circledR}$ pressure sensors, which are depicted in Fig. 12 for reference. It is important to obtain accurate solutions for surface PSD distributions because these are used as input to noise propagation codes for predicting the farfield acoustics. The computed PSD distributions from this study at two locations on the door corresponding to Kulite ${ }^{\circledR}$ pressure sensors 3 and 10 are compared with the experimental data in Fig. 13. Comparisons for sensors 7 (wheel) and 15 (lower torque arm) are presented in Fig. 14. In general, the PSD levels increase with grid refinement and the computational results on finer grids compare favorably with the experimental data, except at the sensor 7 on the wheel. It is not clear if such a discrepancy is caused by difficulty in establishing the exact location of the sensor, or because of the highly sensitive nature of the flow field in the vicinity of this sensor. Solutions from the 24 million node grid indicate much lower PSD levels, and are the least accurate, especially at sensors 7 (wheel) and 15 (lower torque arm). The biggest improvement in predicted levels of PSD is seen going from 24 to 62 million nodes, and the improvement extends to higher frequencies for 142 million node results. The differences in the PSD levels on the 104 million node wall function grid and the 142 million node conventional grid are very minor.

\section{A. Farfield Noise}

Zawodny et al. ${ }^{25}$ have conducted aeroacoustic testing of the PDCC Gulfstream aircraft nose landing gear configuration that is analyzed in this paper. The experiment was performed in the UFAFF, an open jet anechoic tunnel, and a series of 11 microphones were placed at a distance of approximately 7.5 wheel diameters below the wheels along the flyover direction as shown in Fig. 15 (a). Acoustic measurements were also made using 9 microphones placed to the side of this model (see Fig. 15 (b)) at a distance of 5.7 wheel diameters from the centerline. The measured spectra at the microphone locations were corrected to account for shear layer refraction. Note that the effective position of microphone 7 shown in Fig. 15 (a) is directly underneath the nose gear, which closely represents an overhead flyover position of $90^{\circ}$.

We have computed the farfield noise with the conventional hybrid CFD/Acoustic Analogy Approach, where the pressure fluctuations on the solid surfaces of the landing gear configuration obtained from the CFD solutions were used as input to the Ffowcs Williams-Hawkings ${ }^{30}$ (FW-H) solver PSU-WOPWOP ${ }^{31,32}$ for computing the 1/3 octave sound pressure levels (SPL) in dB at farfield points. The results obtained in this manner at flyover microphones 4, 7, and 9 from the 24, 62 and 142 million node grid simulations are shown for a frequency range of 300 to $8000 \mathrm{~Hz}$ in Figs. 16(a)-(c), respectively. These FW-H computations include the contribution of all the pertinent solid surfaces, i.e., the gear, fuselage and mounting plate. The experimental data from the UFAFF, which represents the noise levels at these locations from all the sources for this configuration, is shown as solid black lines connecting filled circles. As was observed for the PSD levels, the overall farfield noise level increases with grid refinement, resulting in improved agreement of the numerical solutions with the experimental data. Keeping in mind that the experimental data at very low frequencies may be corrupted by facility noise, the 142 million node numerical solutions presented here are considered to be in very good agreement with the measurements up to a frequency of $4-5 \mathrm{kHz}$, and the 104 million node wall function solutions closely track these solutions.

The farfield noise results for sideline microphones 3, 5, 7 and 9 are presented in Figs. 17 (a)-(d), respectively. The computational results obtained on the 24, 62 and 142 million node grids suitable for integrating the governing equations to the solid surfaces are compared with the solution on the 104 million node wall function grid, and with the experimental data. The sign convention and notation for symbols and lines used for these figures is identical to the convention used in Figs. 16(a)-(c) for the flyover microphones. Once again, the predicted farfield noise level increases with grid refinement, especially at higher frequencies. The biggest increase in noise level is observed in going from 24 to 62 million nodes, with the predictions on the finest (142 million node) grid being closest to the experimental data up to a frequency of about $4-5 \mathrm{kHz}$, beyond which the predicted noise levels decay very rapidly.

Note that the results on the 104 million node wall function grid closely replicate the results on the 142 million node to within about $1 \mathrm{~dB}$ for both flyover and sideline microphone locations. Because there is very little overhead associated with the wall-function implementation in FUN3D, the reduced node count associated with wall function grids results in significant savings in computational costs to obtain solutions of comparable accuracy for aeroacoustic problems of practical interest.

\section{B. Beamform Maps}

Beamform (or source localization) maps can be used to further analyze the acoustic signals by identifying the location and relative strengths of prominent noise sources. Zawodny et al. ${ }^{25}$ acquired farfield noise measurements in the UFAFF with a phased array of 64 microphones that was placed along the flyover and sideline planes of the landing gear 
configurations. A top view of the flyover array is shown in Fig. 18, which is placed approximately 8 wheel diameters below the wheels. This phased microphone array is placed in a quiescent region outside the shear layers induced by the open jet of the UFAFF tunnel. The results from the beamform analysis are presented here along the flyover direction, which is considered important from the community noise perspective, since it represents a region directly under the flight path.

For the present analysis, pressure records at the phased array microphone locations were generated using the FW-H approach in conjunction with the computed surface pressures. The PSD levels computed at the center of the microphone array based on the pressure records are compared in Fig. 19 for the 62 million and 142 million node grids. Results obtained from the 104 million node wall function grid are also included in this figure. Computational results for the 62 and 142 million node grids are in good agreement up to a frequency of $3 \mathrm{kHz}$, beyond which the PSD levels on the 142 million node case are slightly higher compared to the 62 million node results. The results from the 104 million node wall function grid are within $1 \mathrm{~dB}$ of the 142 million node results, which is similar to the agreement observed for the farfield noise results presented in Figs. 16-17.

The AVEC Inc. beamforming suite ${ }^{33}$ was used to analyze the synthetic microphone array data. This included standard beamforming processing and the deconvolution based algorithm proposed by Sijtsma. ${ }^{34}$ A typical source localization (beamform) map reconstructed from the unsteady pressure fluctuations at the 64 locations corresponding to the flyover microphone array of Ref. 25 is presented in Fig. 20 at a frequency of $1 \mathrm{kHz}$. The SPL contours presented in these figures are normalized such that a value of 0 corresponds to the local maximum for each set, and lower SPL levels are represented by negative values. Such a representation is very useful to compare results from different grids or methods in terms of identifying the noise sources and their relative strengths, even though the absolute values of noise levels are not given on these plots. Results from the 62 million node grid are compared with those obtained on the 142 million node grid in Fig. 20. The maps from both sets of computations are very similar, and identify the gear door as the biggest source of noise at this frequency. A slight asymmetry relative to the model centerline is observed in these contour maps. Most probably, this is due to the unsteady shedding caused by the slight asymmetry of connecting rods (struts) and gear door surface geometries in the gear assembly, and a lack of symmetry in the grids used here.

Similar plots at $3 \mathrm{kHz}$ and $5 \mathrm{kHz}$ frequencies are shown in Figs. 21 and 22, respectively. The computed noise source locations on both grids compare well with each other, and indicate a forward movement of the primary noise source at higher frequencies. At a frequency of $3 \mathrm{kHz}$, the primary source of noise is associated with the torque arm for both of these grids, as seen in Fig. 21. Another significant, although weaker noise source is also visible near the gear door at this frequency. As observed in Fig. 22, the location of the dominant noise sources in the vicinity of wheel axle and torque arm at the higher frequency of $5 \mathrm{kHz}$ is nearly identical to those observed at the frequency of $3 \mathrm{kHz}$. However, the noise source on the door moves upstream closer to the wheels at $5 \mathrm{kHz}$. In addition, several other much weaker sources are observed at the edges of the wheels and the gear door at this higher frequency. Based on these results, the gear door appears to be the most dominant noise source at low frequencies, with the torque arm being the most dominant source at higher frequencies above $1 \mathrm{kHz}$. Although not shown here, the results from 104 million node wall function grid are nearly identical to the 142 million node grid results.

\section{Concluding Remarks}

A systematic grid refinement study is presented here for computing unsteady solutions for turbulent viscous flow over a realistic nose landing gear configuration with a hybrid RANS/LES approach. Unexpected difficulties were encountered in initial phases of this work when nonphysical results were obtained on the finest grid with the SA-based DDES model. After careful examination of the behavior of shielding function on finer grids, a simple fix was suggested to eliminate such anomalous behavior by dropping the $f_{t 2}$ term from the base turbulence model.

Mixed-element grids used in this work were generated with the Pointwise ${ }^{\circledR}$ grid generation code. This approach is found to be very robust and efficient for generating a family of successively finer grids that are suitable for conducting grid refinement studies for aeroacoustic simulations. By simply changing a few parameters, a set of uniformly refined grids consisting of 24, 62 and 142 million nodes were generated with this approach. In addition, a grid suitable for wall function approach consisting of 104 million nodes and a nominal value of $y^{+}=25$ was generated such that it replicated the surface grid distribution of the 142 million node grid. The off-surface grid distribution of the 104 million node grid beyond the boundary layer regions was also comparable to the 142 million node grid.

Computed time-averaged pressure and turbulence kinetic energy distributions from the 24, 62 and 142 million node grids are shown to be in good agreement with experimental data and previous results published for this configuration. The biggest improvement in accuracy is observed going from 24 to 62 million nodes. The results from the 104 million node wall function grid are nearly identical to the 142 million node grid results. 
Surface PSD levels computed from the current unsteady flow solutions are compared with the experimental data at selected sensor locations. In general, the solution accuracy improves with grid refinement. In particular, the solution accuracy on the current 62 million node grid is found to be quite good, and comparable to previously published results on much larger grids. Further improvement in accuracy is observed at higher frequencies on the 142 million node grid. Similar observations are made for the farfield noise at selected microphone locations. Results obtained on the 104 million node wall function grid for the PSD and farfield noise levels differ from the 142 million node results by no more than than $1 \mathrm{~dB}$ at the same locations. Because the computational cost varies linearly with node count, significant savings (e.g. $40 \%$ savings for this case) can be achieved by making use of wall functions to obtain comparable accuracy for such simulations. Finally, it is concluded that the gear door is the biggest noise source at the lower frequencies $(<1 \mathrm{kHz})$, whereas the torque arm is the most dominant noise source at the higher frequencies.

\section{Acknowledgments}

This work was supported by NASA Advanced Air Vehicles Program through the Advanced Air Transportation Technology Project. The authors would also like to acknowledge Mr. Michael Wiese of Craig Technologies, Inc. for generating the grids used in this work.

\section{References}

\footnotetext{
${ }^{1}$ Dobrzynski, W., "Almost 40 years of Airframe Noise Research: What Did We Achieve," Journal of Aircraft, Vol. 47, No. 2, 2010 , pp. $353-367$.

${ }^{2}$ Vuillot, F., Housen, F., Manoha, E., Redonnet, S., and Jacob, J., "Application of the CEDRE unstructured flow solver to landing gear unsteady flow and noise predictions," AIAA Paper 2015-2944, June 2015.

${ }^{3}$ De La Puente, F., Sanders, F., Vuillot, F., and Manoha, E., "Nose landing gear flow and noise predictions on unstructured grid using a cellcentered Navier-Stokes code," AIAA Paper 2015-3256, June 2015.

${ }^{4}$ Heffernon, T., Angland, D., Zhang, X., and Smith, M., "The effect of flow circulation on the Scattering of Landing Gear Noise," AIAA Paper 2015-3257, June 2015.

${ }^{5}$ Bpuvy, Q., Rougier, T., Ghouali, A., Casalino, D., Appelbaum, J., and Kleinclaus, C., "Design of quieter landing gears throgh lattice-Boltzmann CFD simulations," AIAA Paper 2015-3259, June 2015.

${ }^{6}$ Vatsa, V. N., Lockard, D. P., and Khorrami, M. R., "Application of FUN3D Solver for Aeroacoustics Simulation of a Nose Landing Gear Configuration," AIAA Paper 2011-2820, June 2011.

${ }^{7}$ Vatsa, V. N., Lockard, D. P., Khorrami, M. R., and Carlson, J.-R., "Aeroacoustic Simulation of a Nose Landing Gear in an Open Jet Facility using FUN3D," AIAA Paper 2012-2280, June 2012.

${ }^{8}$ Biedron, R. T., Carlson, J.-R., Derlaga, J. M., Gnoffo, P. A., Hammond, D. P., Jones, W. T., Kleb, W., Lee-Rausch, E. M., Nielsen, E. J., Park, M. A., Rumsey, C. L., Thomas, J. L., and Wood, W. A., "FUN3D Manual: 12.7,” NASA TM 2015-218761, May 2015.

${ }^{9}$ FUN3D Web page: http://fun3d.larc.nasa.gov, 2015 (accessed April 2016).

${ }^{10}$ Vatsa, V. N. and Lockard, D. P., "Assessment of Hybrid RANS/LES Turbulence Models for Aeroacoustics Applications," AIAA Paper 20104001, June 2010.

${ }^{11}$ Rumsey, C., Biedron, R., and Thomas, J., “CFL3D: Its History and Some Recent Applications,” NASA TM 112861, May 1997, presented at the Godonov's Method for Gas Dynamics Symposium, Ann Arbor, MI.

${ }^{12}$ Pirzadeh, S., "Three-dimensional Unstructured Viscous Grids by the Advancing Layer Method," AIAA Journal, Vol. 33, No. 1, 1996, pp. 43-49.

${ }^{13}$ Vatsa, V. N., Khorrami, M. R., Park, M. A., and Lockard, D. P., "Aeroacoustic Simulation of Nose Landing Gear on Adaptive Unstructured Grids with FUN3D,” AIAA Paper 2013-2071, May 2013.

${ }^{14}$ Vatsa, V. N., Khorrami, M. R., Rhoads, J., and Lockard, D. P., "Aeroacoustic Simulation of a Nose Landing Gear using FUN3D on Pointwise Unstructured Grids," AIAA Paper 2015-3255, June 2015.

${ }^{15}$ Lynch, C. and Smith, M. J., "Hybrid RANS-LES Turbulence Models on Unstructured Grids," AIAA Paper 2008-3854, June 2008.

${ }^{16}$ Baurle, R. and Edwards, J., “Hybrid Reynolds-Averaged/Large-Eddy Simulations of a Coaxial Supersonic Free-Jet Experiment,” AIAA Paper 2009-0129, January 2009.

${ }^{17}$ Anderson, W. K. and Bonhaus, D. L., "An Implicit Upwind Algorithm for Computing Turbulent Flows on Unstructured Grids," Computers and Fluids, Vol. 23, No. 1, 1994, pp. 1-21.

${ }^{18}$ Alexandrov, N., Atkins, H. L., Bibb, K. L., Biedron, R. T., Gnoffo, P. A., Hammond, D. P., Jones, W. T., Kleb, W. L., Lee-Rausch, E. M., Nielsen, E. J., Park, M. A., Raman, V. V., Roberts, T. W., Thomas, J. L., Vatsa, V. N., Viken, S. A., White, J. A., and Wood, W. A., "Team Software Development for Aerothermodynamic and Aerodynamic Analysis and Design,” NASA TM 2003-212421, Nov. 2003.

${ }^{19}$ Nielsen, E., Lu, J., Park, M., and Darmofal, D., "An Implicit, Exact Dual Adjoint Solution Method for Turbulent Flows on Unstructured Grids," Computers and Fluids, Vol. 33, No. 9, 2003, pp. 1131-1155.

${ }^{20}$ Roe, P. L., "Approximate Riemann Solvers, Parameter Vectors, and Difference Schemes," Journal of Computational Physics, Vol. 43, No. 2, 1981, pp. 357-372.

${ }^{21}$ Biedron, R. T., Vatsa, V. N., and Atkins, H. L., "Simulation of Unsteady Flows using an Unstructured Navier-Stokes Solver for Moving and Stationary Grids," AIAA Paper 2005-5039, June 2005.

${ }^{22}$ Vatsa, V. N., Carpenter, M. H., and Lockard, D. P., "Re-evaluation of an Optimized Second Order Backward Difference (BDF2OPT) Scheme for Unsteady Flow Applications," AIAA Paper 2010-0122, January 2010.

${ }^{23}$ Spalart, P. R., "Young Person's Guide to Detached-Eddy Simulation Grids,” NASA CR-211032, July 2001.
} 
${ }^{24}$ Spalart, P. R., Deck, S., Shur, M. L., Squires, K. D., Strelets, M. K., and Travin, A., “A New Version of Detached-Eddy Simulation, Resistant to Ambiguous Grid Densities," Theoretical and Computational Fluid Dynamics, Vol. 20, No. 3, 2006, pp. 181-195.

${ }^{25}$ Zawodny, N., Liu, F., Yardibi, T., Cattafesta, L., Khorrami, M., Neuhart, D., and Van de Ven, T., "A Comparative Study of a 1/4-scale Gulfstream G550 Aircraft Nose Gear Model,” AIAA Paper 2009-3153, May 2009.

${ }^{26}$ Neuhart, D., Khorrami, M., and Choudhari, M., "Aerodynamics of a Gulfstream G550 Nose Landing Gear Model,” Journal of Aircraft, Vol. 44, No. 6, 2007, pp. 1769-1775.

${ }^{27}$ Carlson, J.-R., "Inflow/Outflow Boundary Conditions with Application to FUN3D," NASA TM 2011-217181, NASA Langley Research Center, Oct. 2011.

${ }^{28}$ Vatsa, V., Lockard, D., and Spalart, P., "Grid sensitivity of the SA-based Delayed Detached Eddy Simulation Model for Blunt Body Flows," Accepted for publication in AIAA Journal, March 2017.

${ }^{29}$ Turbulence Modeling Resource Web page: http://turbmodels.larc.nasa.gov, 2016 (accessed March 2017).

${ }^{30}$ Ffowcs Williams, J. E. and Hawkings, D. L., "Sound Generated by Turbulence and Surfaces in Arbitrary Motion," Philosophical Transactions of the Royal Society, Vol. A264, No. 1151, 1969, pp. 321-342.

${ }^{31}$ Brentner, K. S., Lopes, L. V., Chen, H. N., and Horn, J. F., "Near Real-Time Simulation of Rotorcraft Acoustics and Flight Dynamics," 59th Annual Forum, AHS International, Alexandria, VA, 2003.

${ }^{32}$ Bres, G. A., Brentner, K. S., Perez, G., and Jones, H. E., "Maneuvering Rotorcraft Noise Prediction," Journal of Sound and Vibration, Vol. 275 , No. 3-5, 2004, pp. 719-738.

${ }^{33}$ AVEC Web page: http://www.avec-engineering.com/products.html, 2015 (accessed March 2017).

${ }^{34}$ Sijtsma, P., "CLEAN Based on Spatial Source Coherence," AIAA Paper 2007-3436, May 2007. 


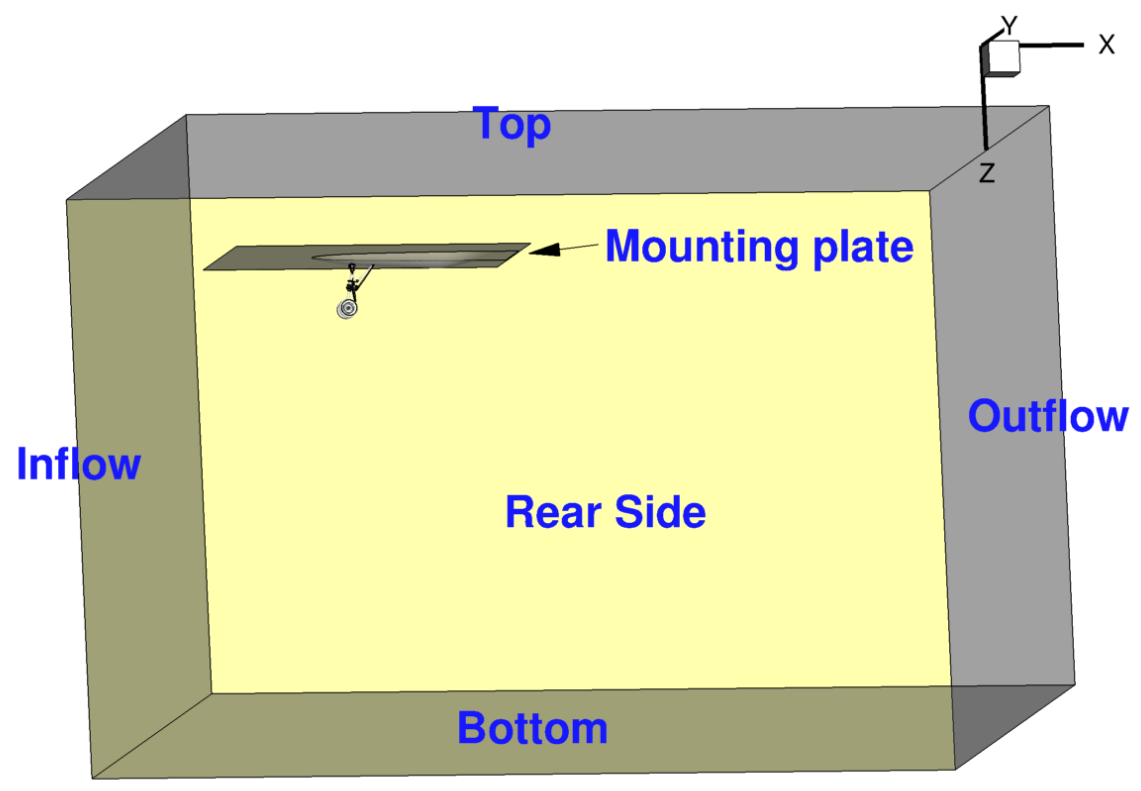

Figure 1. Computational model of the nose landing gear in UFAFF tunnel.

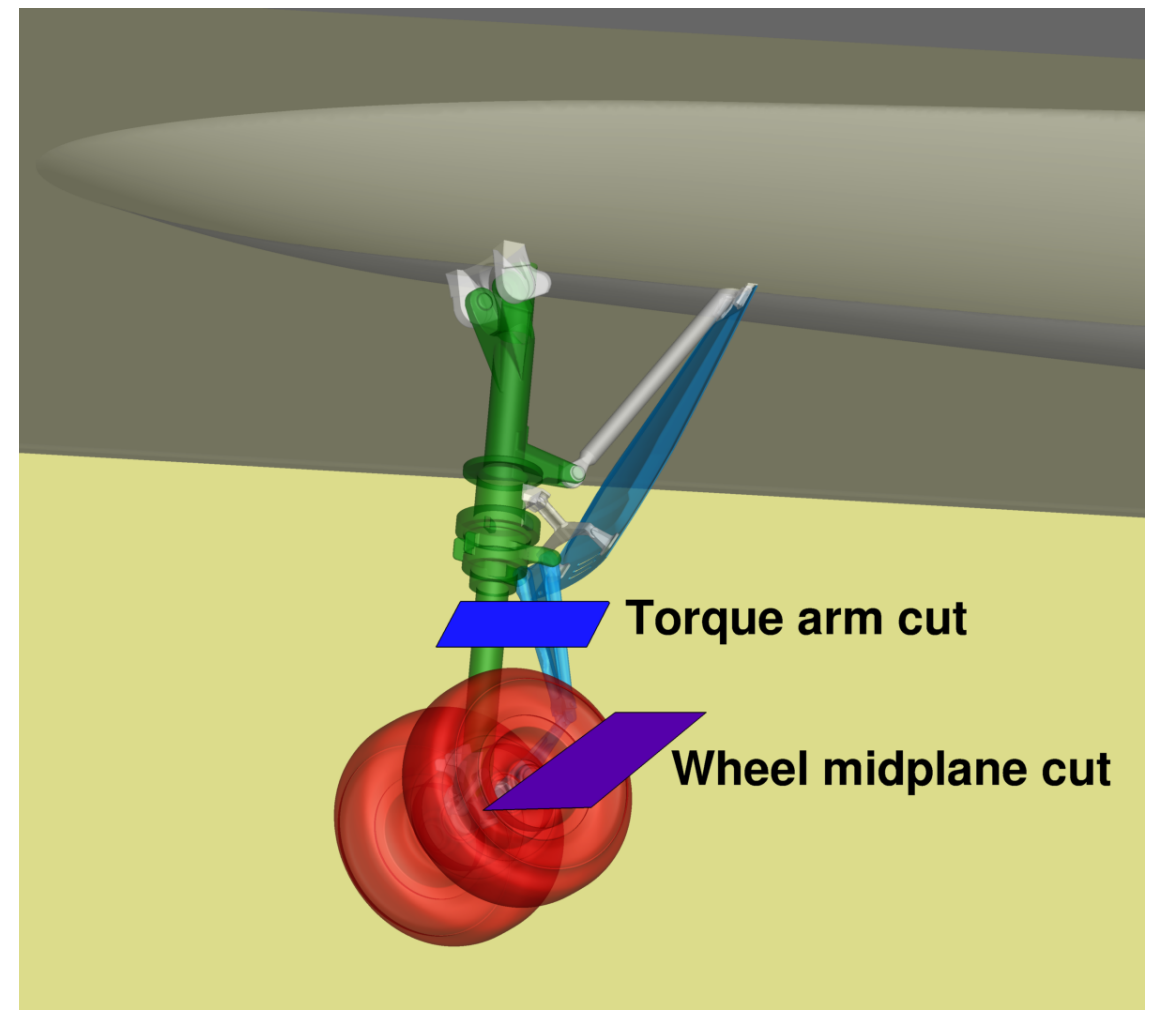

Figure 2. Wheel and torque arm cut locations. 


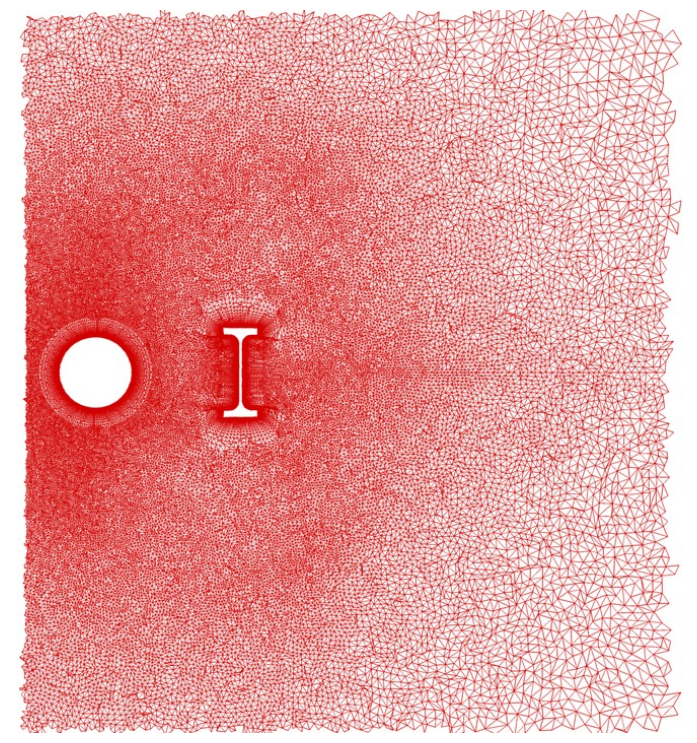

(a) $24 \mathrm{M}$ nodes

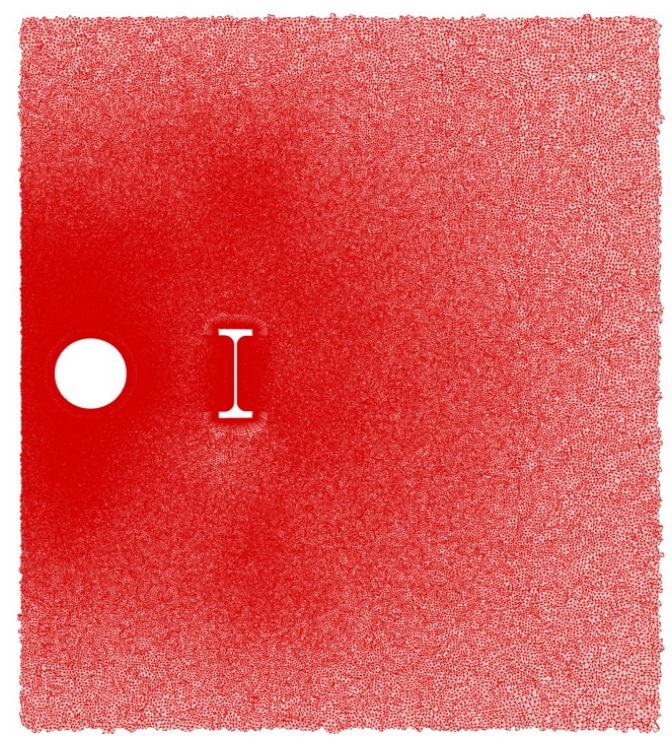

(c) 142M nodes

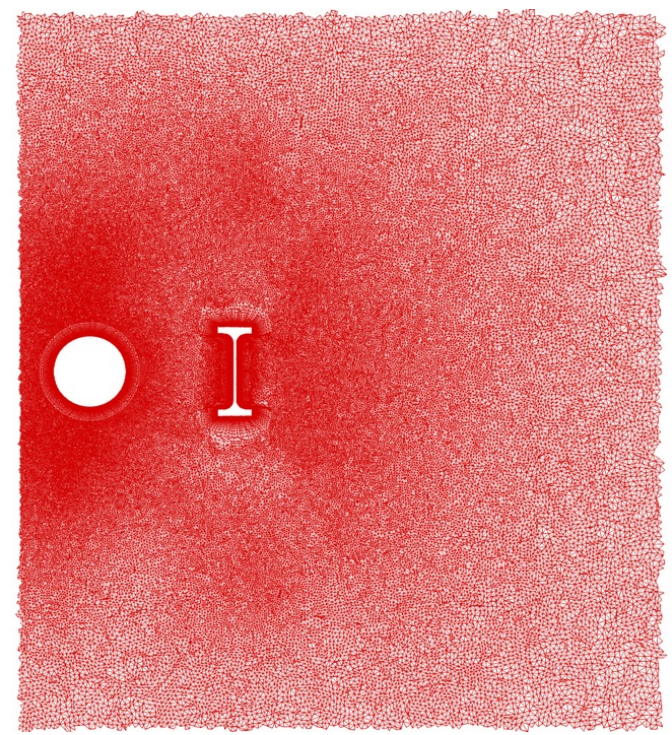

(b) 62M nodes

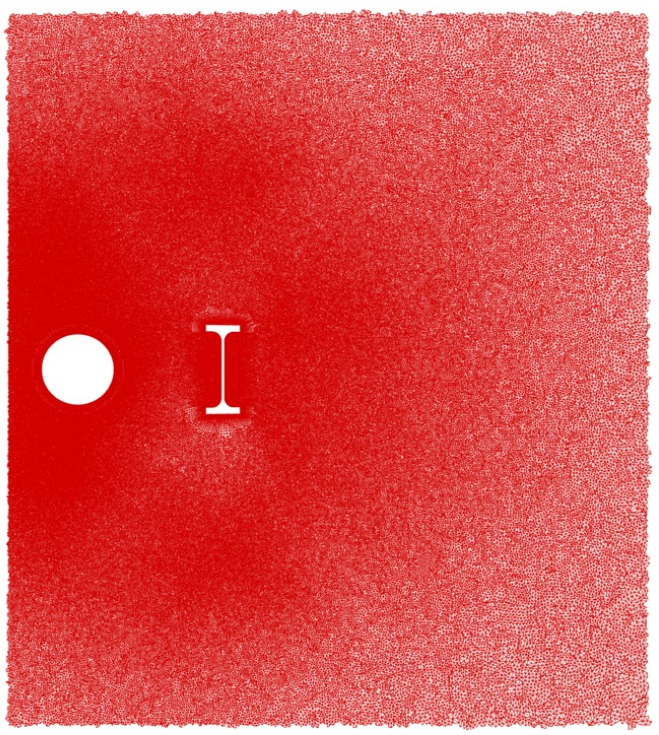

(d) 104M nodes $\left(y^{+}=25\right)$

Figure 3. Comparison of mesh distribution at torque arm cut. 


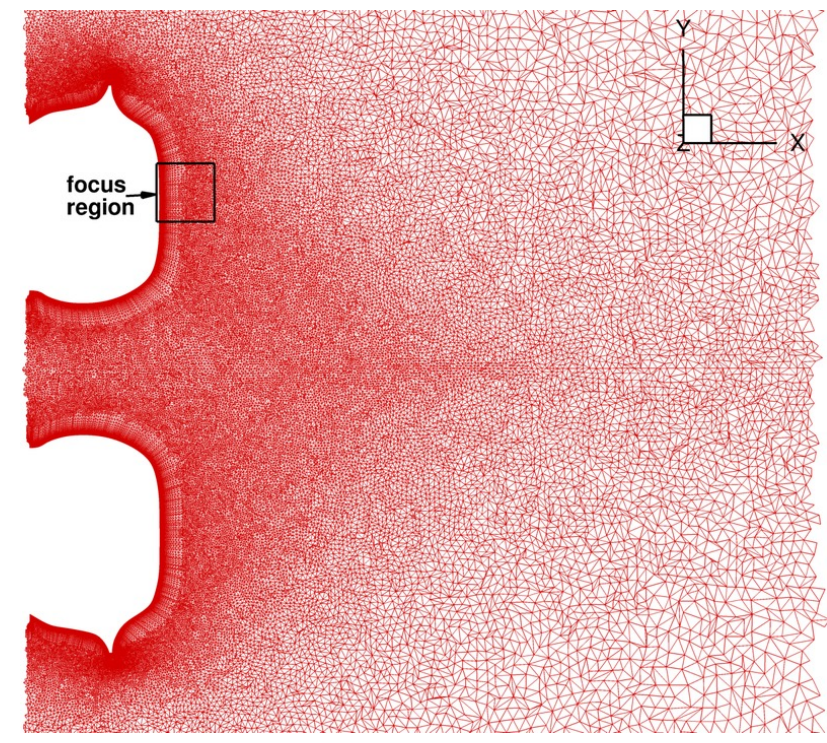

(a) 24M nodes

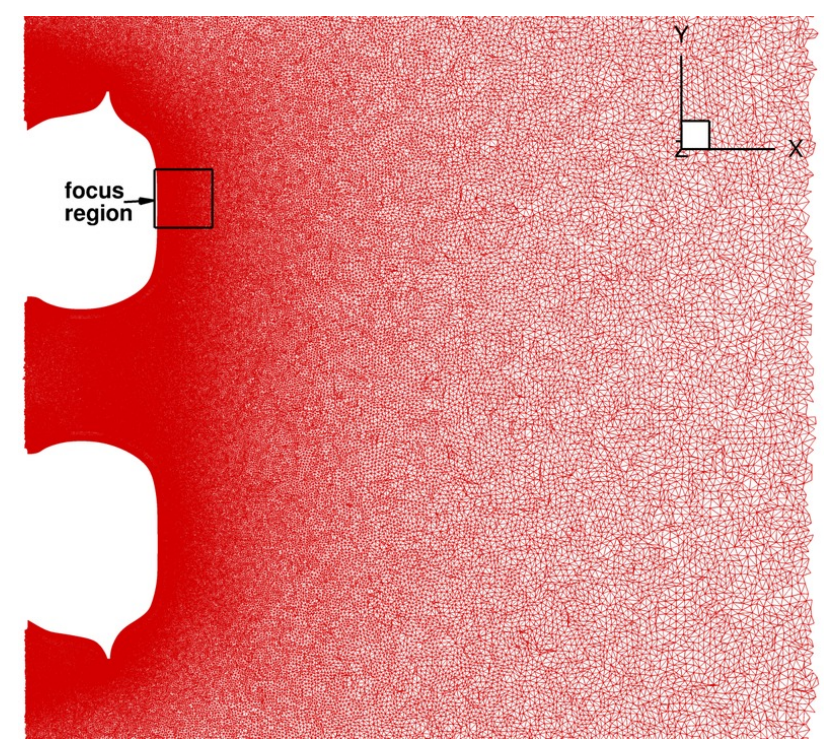

(c) $142 \mathrm{M}$ nodes

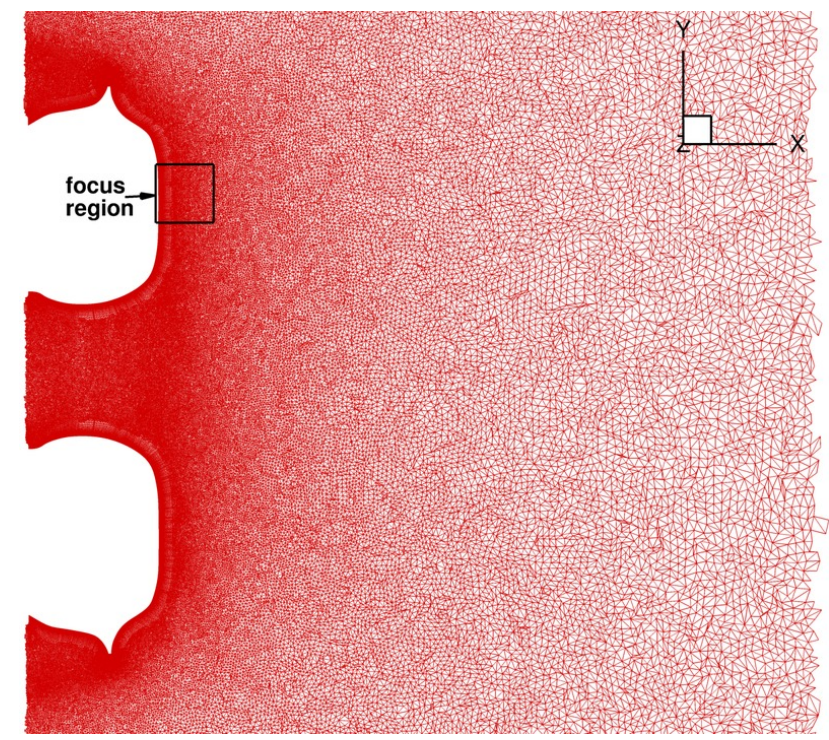

(b) 62M nodes

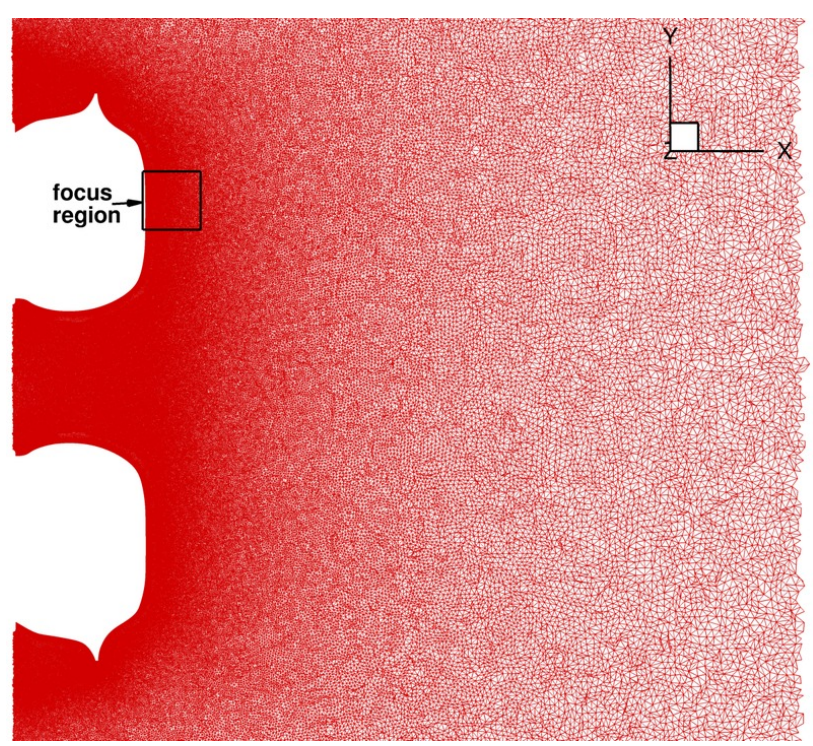

(d) $104 \mathrm{M}$ nodes $\left(y^{+}=25\right)$

Figure 4. Comparison of mesh distribution at midsection of wheel wake, global view. 


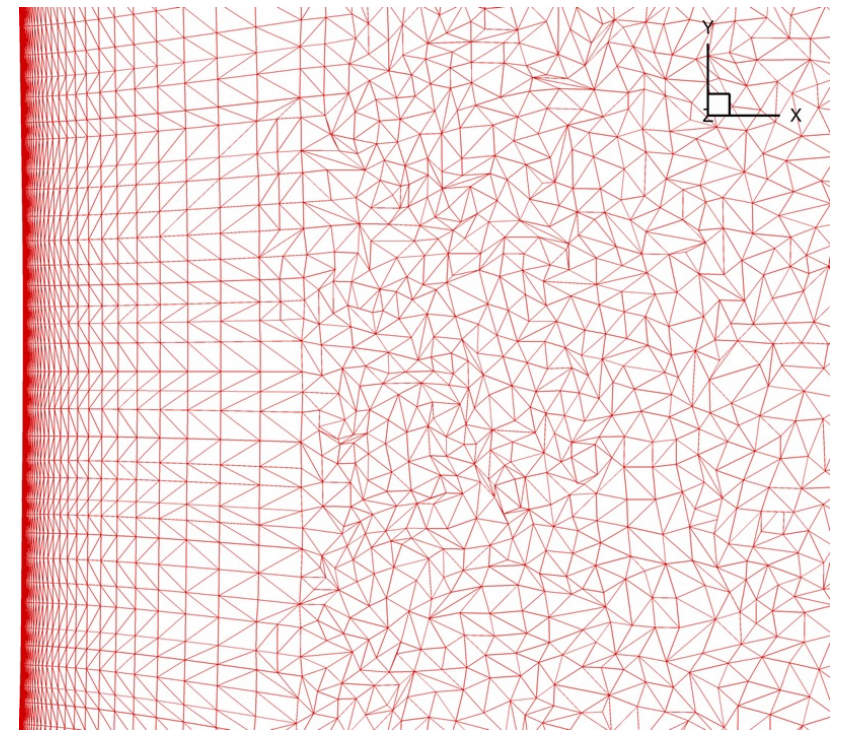

(a) 24M nodes

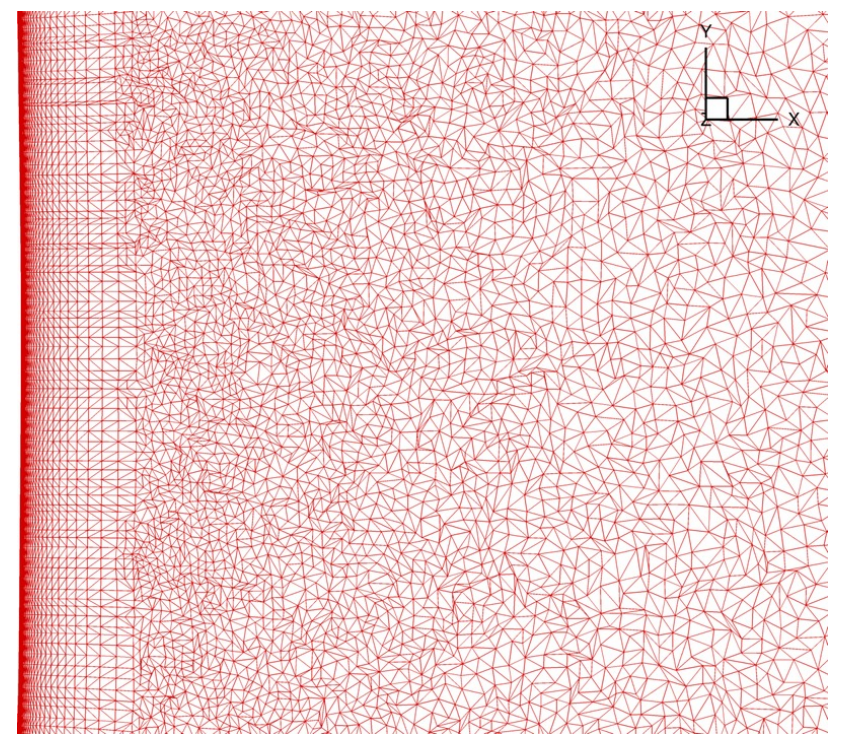

(c) $142 \mathrm{M}$ nodes

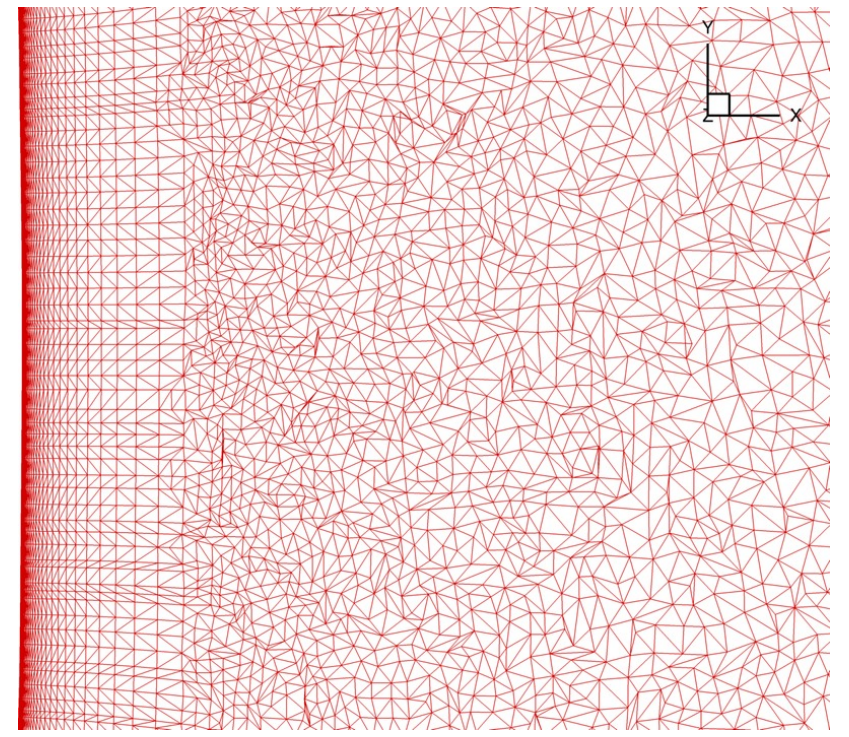

(b) 62M nodes

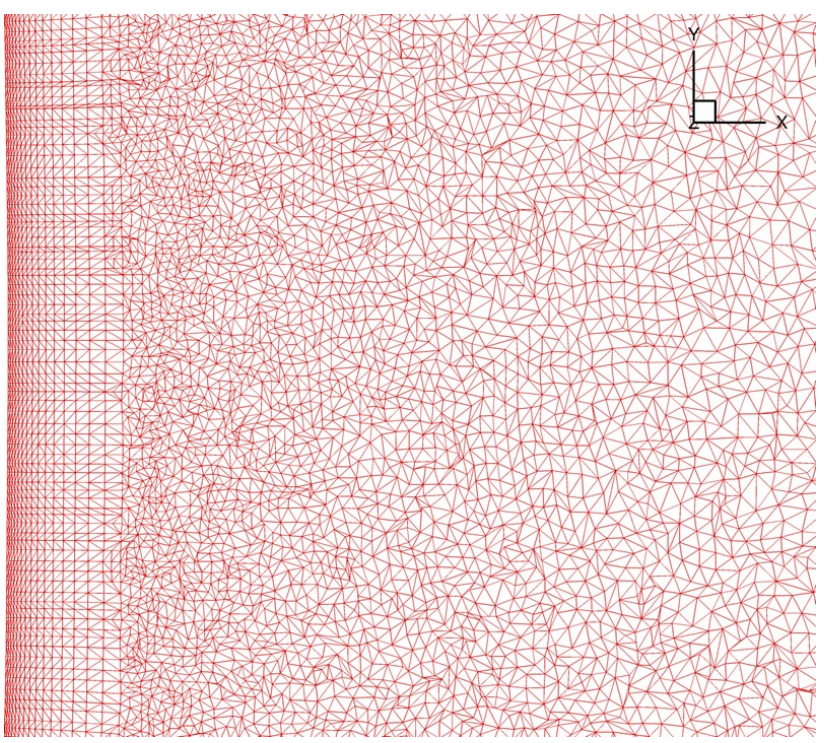

(d) 104M nodes $\left(y^{+}=25\right)$

Figure 5. Comparison of mesh distribution at midsection of wheel wake, focus region. 


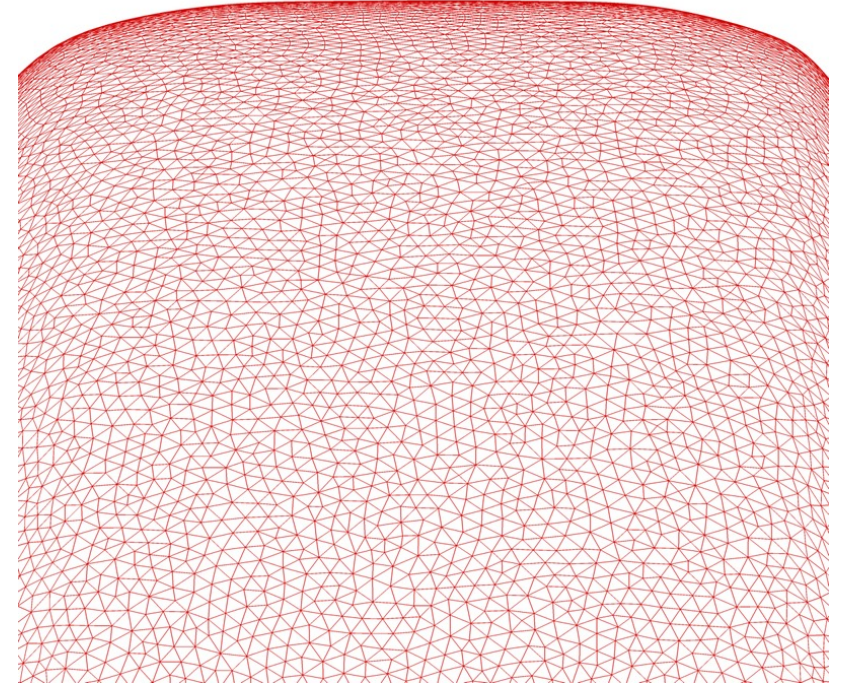

(a) $24 \mathrm{M}$ nodes

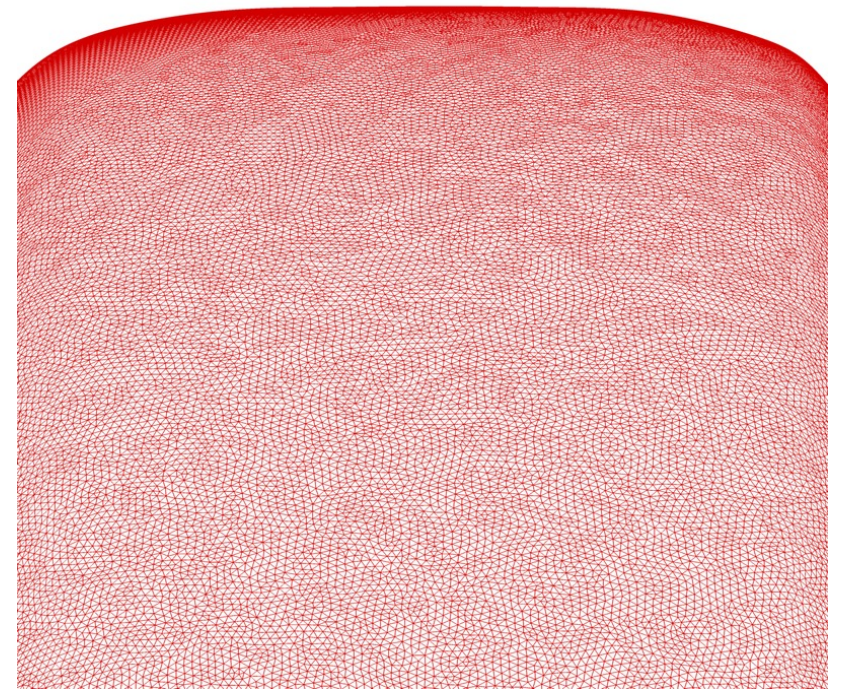

(c) 142M nodes

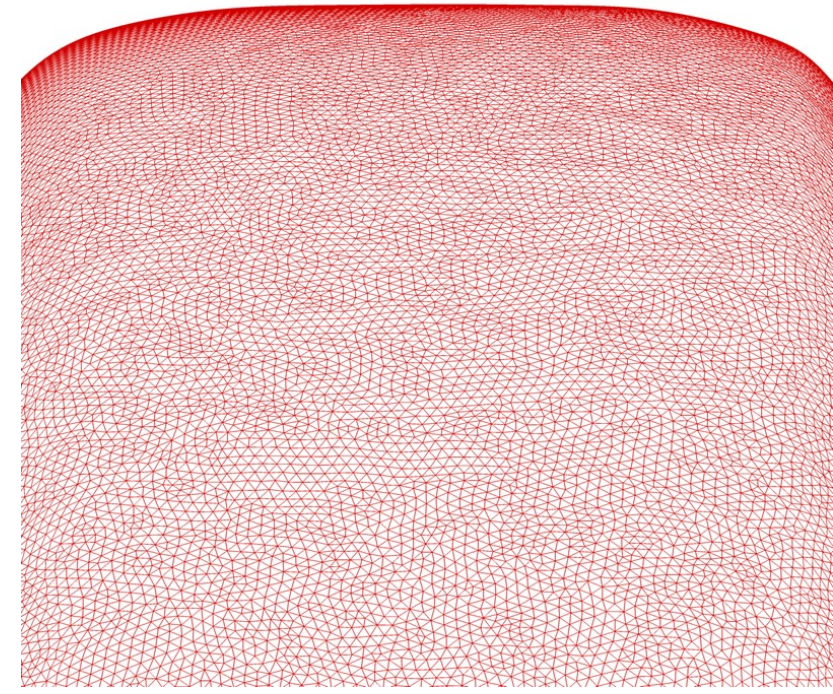

(b) 62M nodes

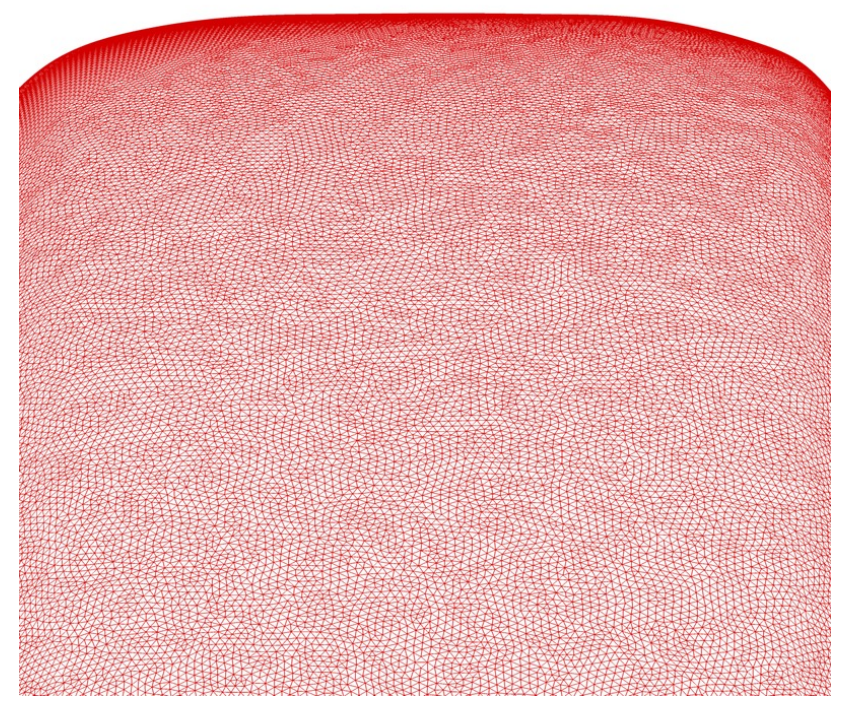

(d) $104 \mathrm{M}$ nodes $\left(y^{+}=25\right)$

Figure 6. Comparison of surface mesh distribution at midsection of port wheel. 


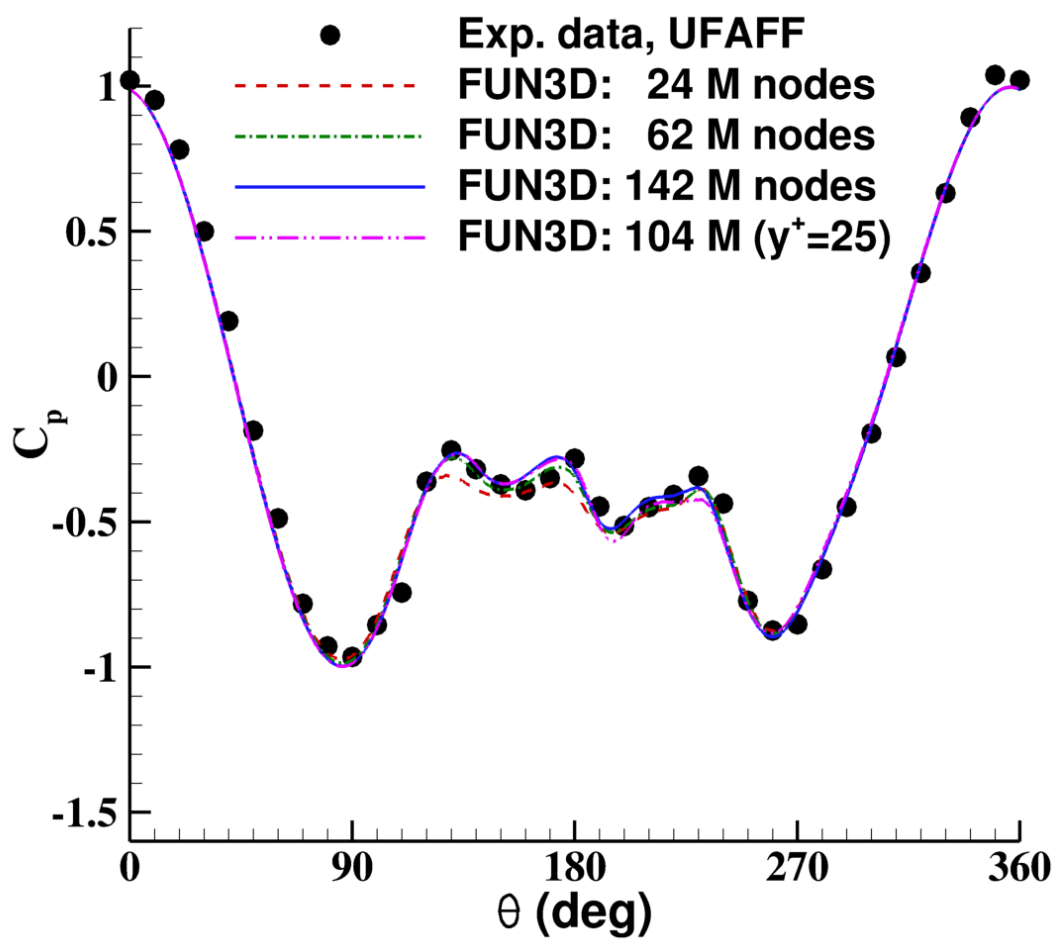

Figure 7. Surface pressure distributions on port wheel. 


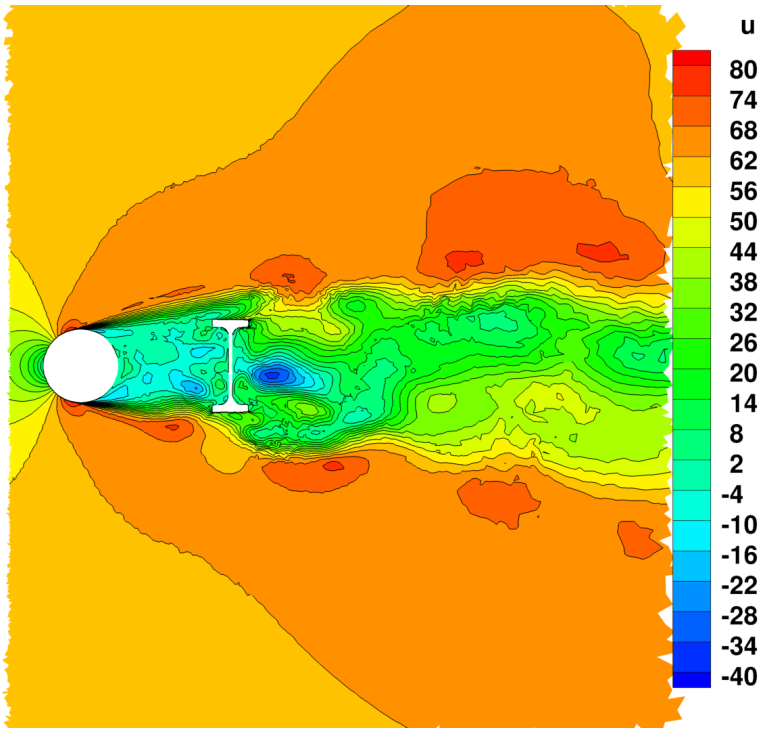

(a) $24 \mathrm{M}$ nodes

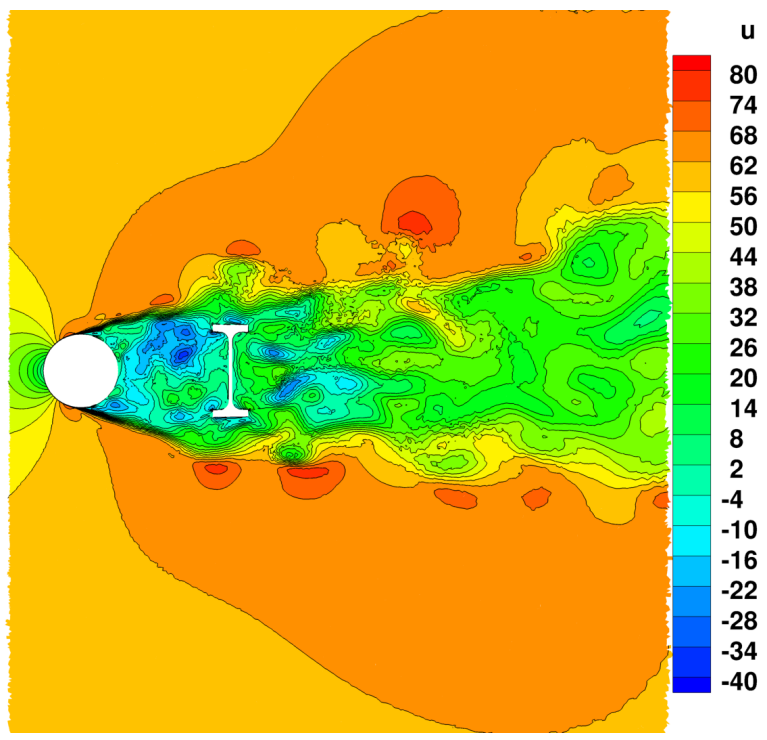

(c) 142M nodes

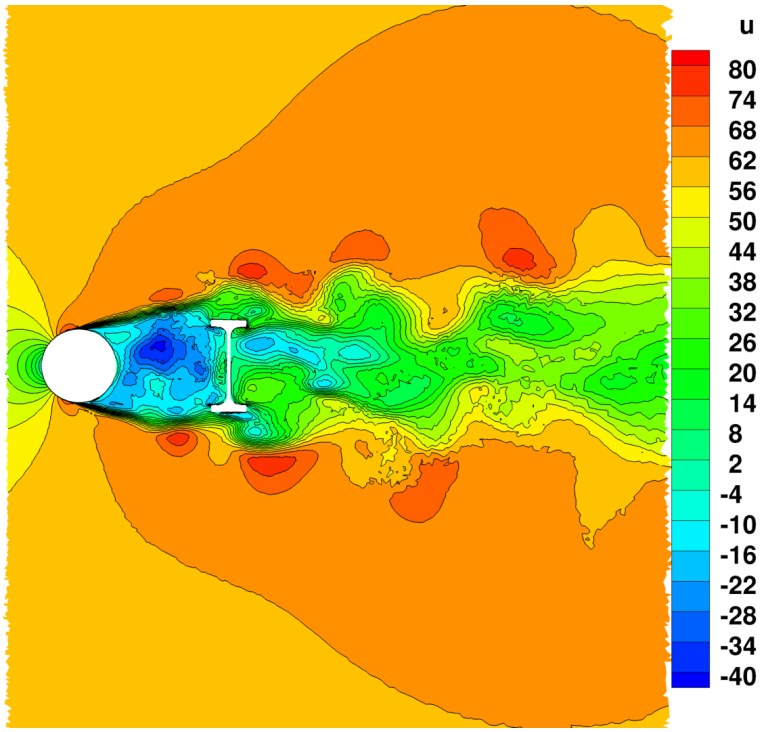

(b) 62M nodes

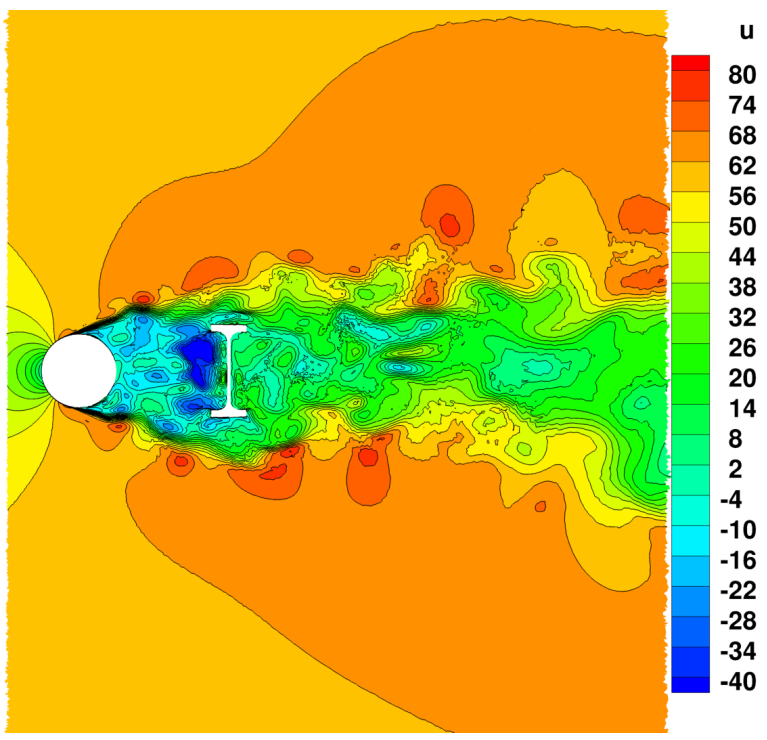

(d) 104M nodes $\left(y^{+}=25\right)$

Figure 8. Comparison of instantaneous velocity (meter/second) contours at torque arm cut. 


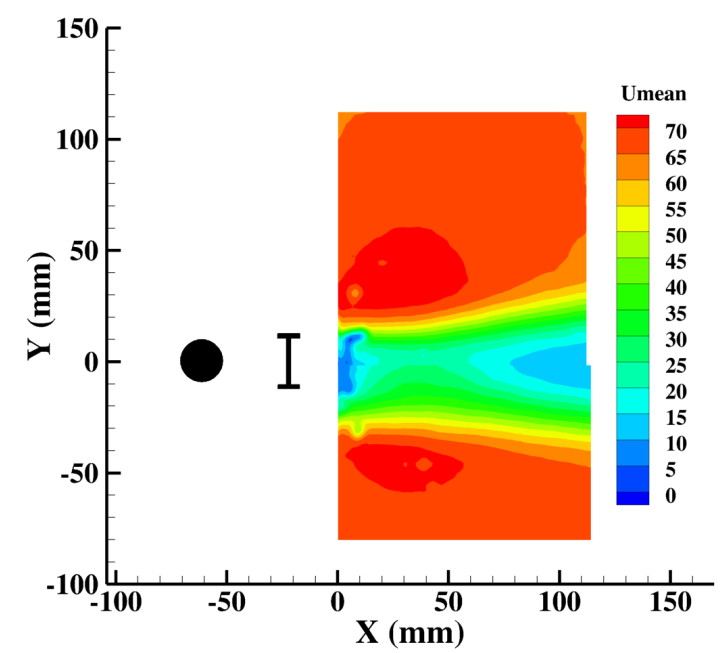

(a) Exp. PIV data

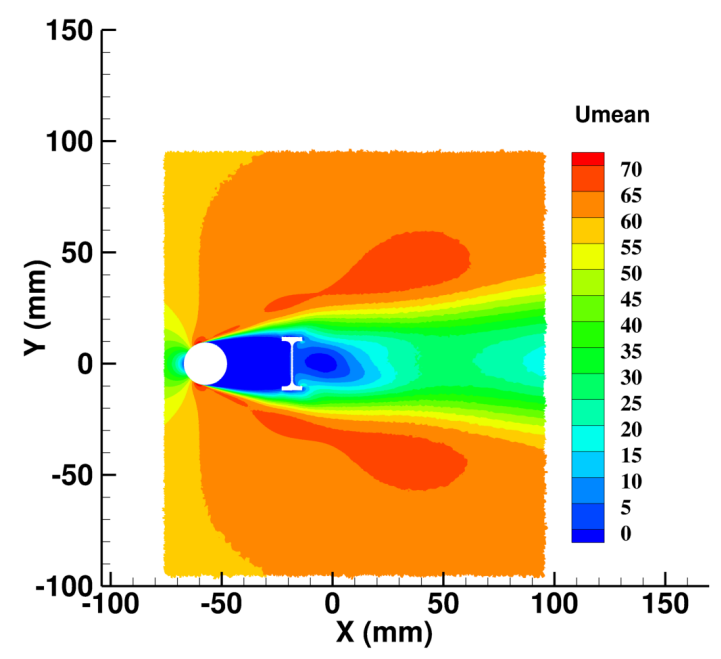

(c) FUN3D:142M nodes

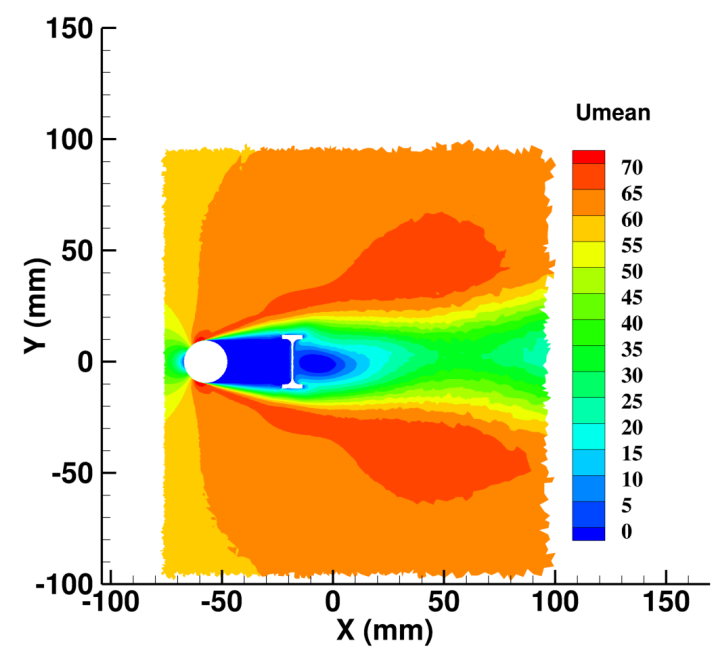

(b) FUN3D: 24M nodes

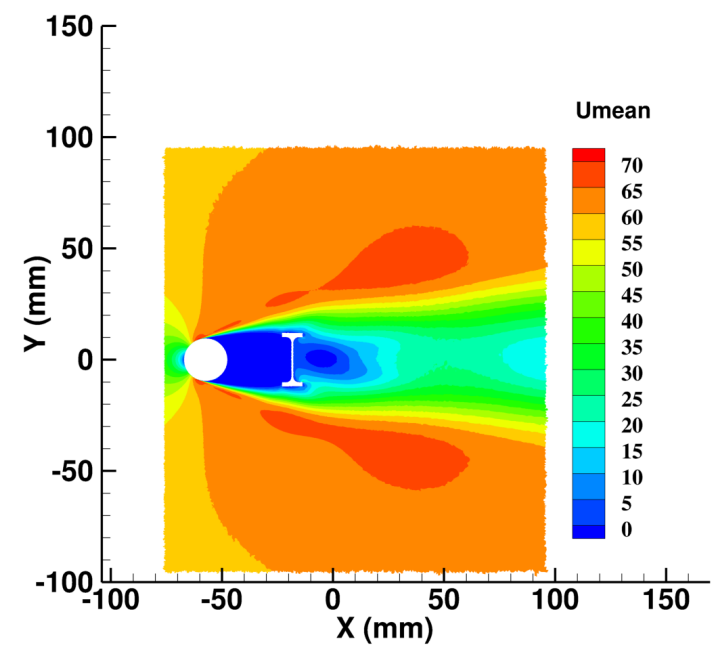

(d) FUN3D:104M nodes $\left(y^{+}=25\right)$

Figure 9. Comparison of time-averaged streamwise velocity (meter/second) contours at torque arm cut. 


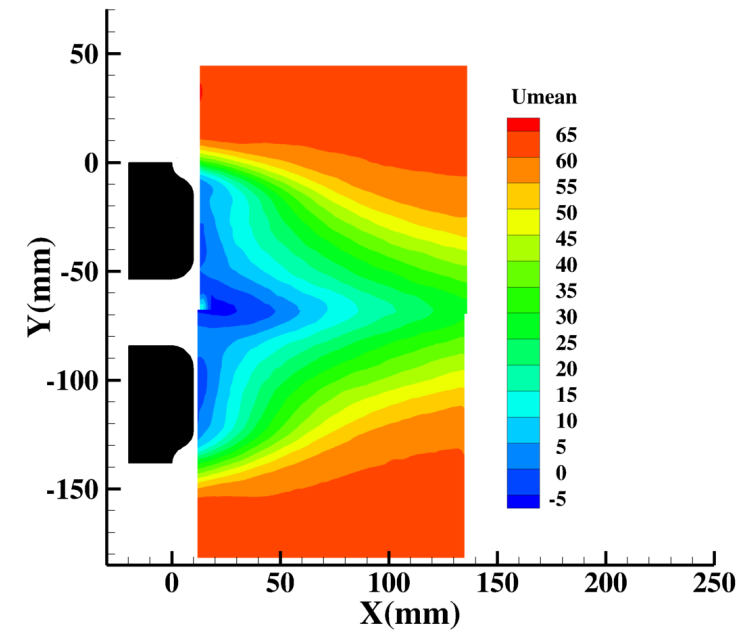

(a) Exp. PIV data

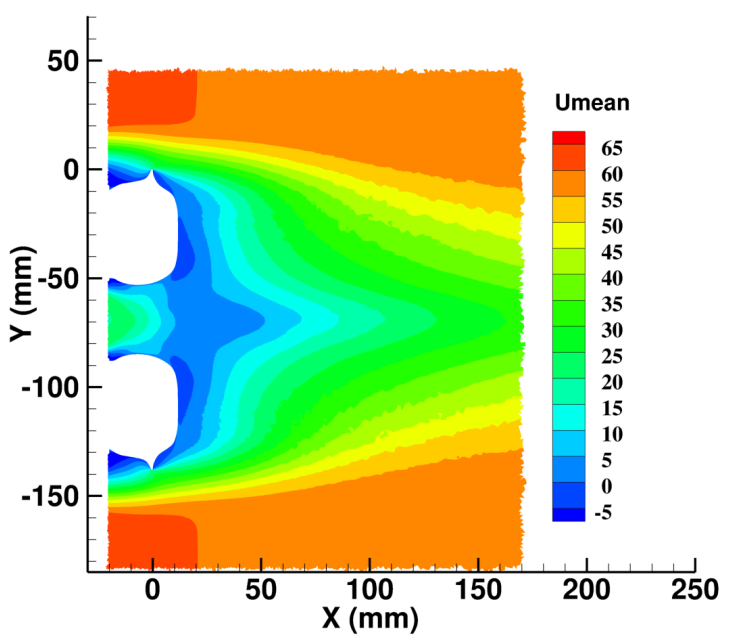

(c) FUN3D:142M nodes

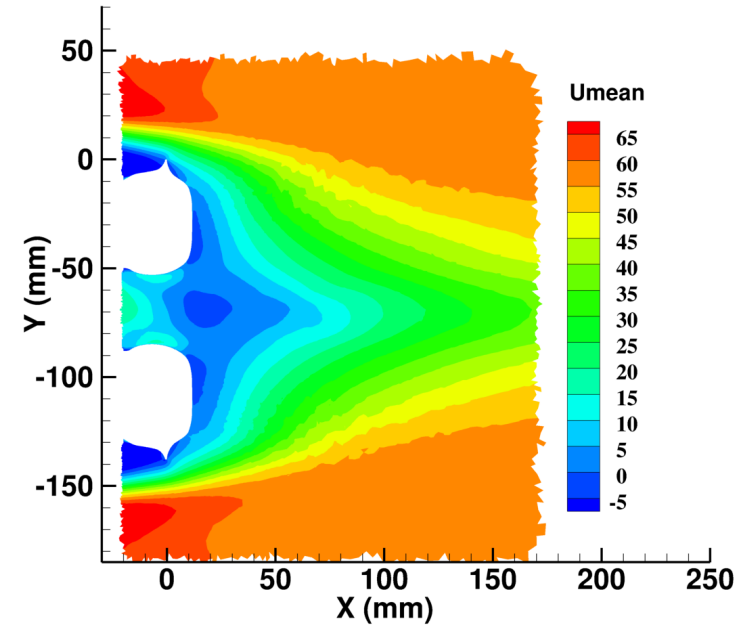

(b) FUN3D: 24M nodes

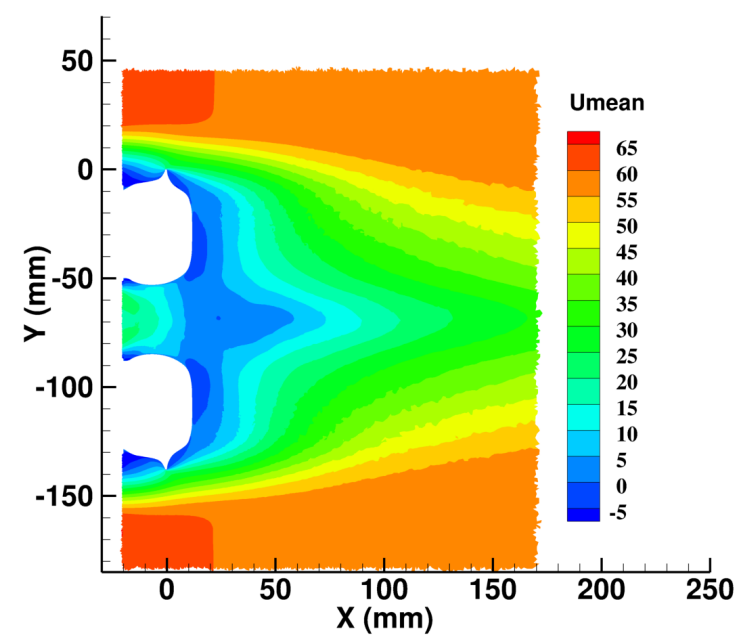

(d) FUN3D:104M nodes $\left(y^{+}=25\right)$

Figure 10. Comparison of time-averaged streamwise velocity (meter/second) contours, midwheel plane cut. 


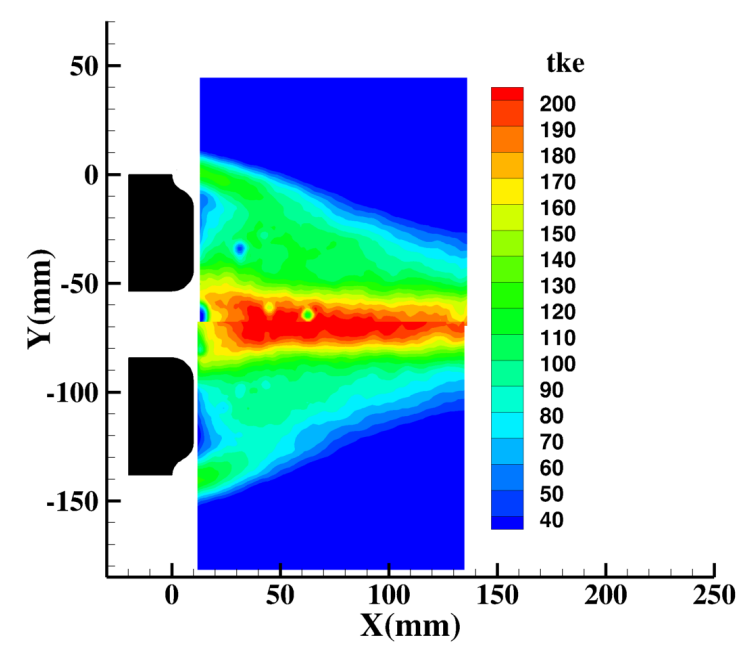

(a) Exp. PIV data

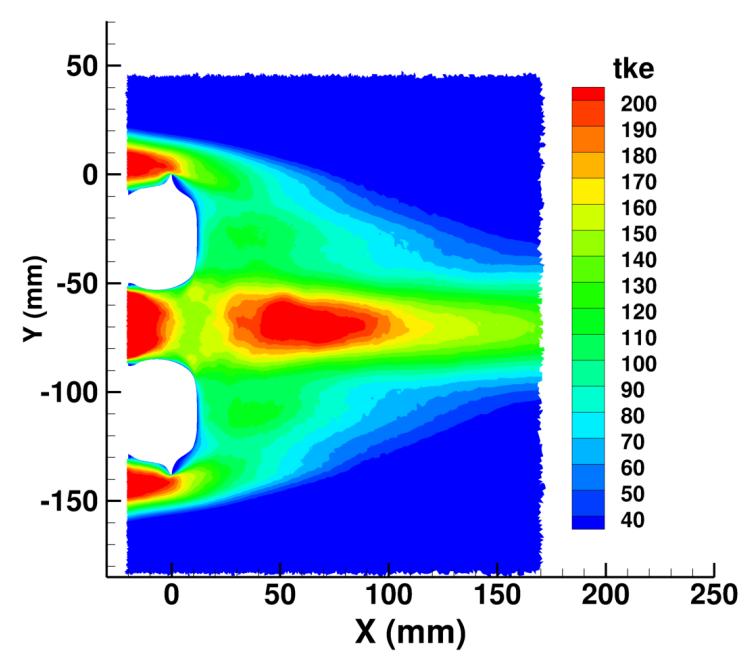

(c) FUN3D:142M nodes

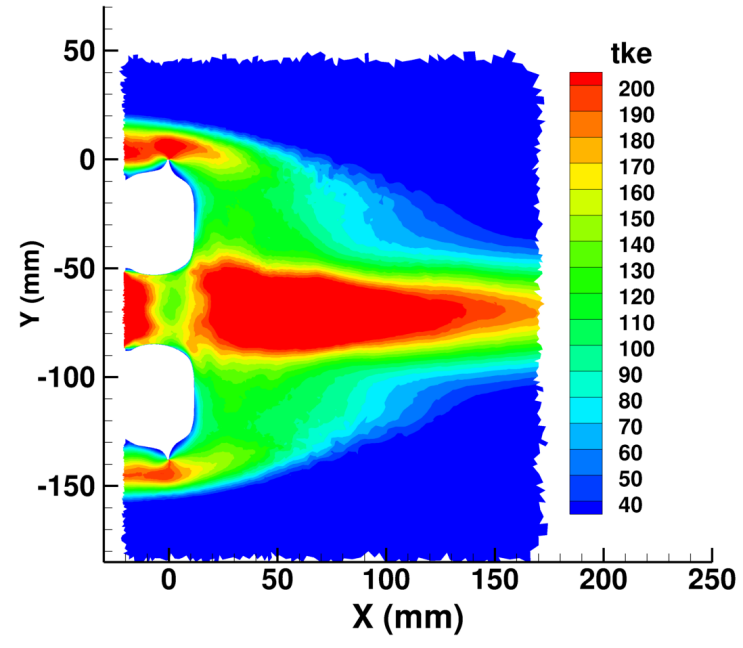

(b) FUN3D: 24M nodes

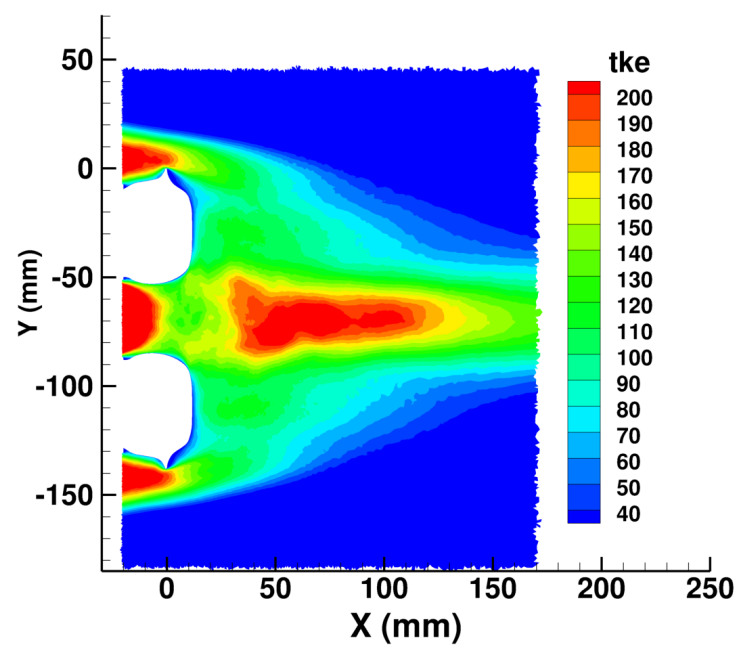

(d) FUN3D:104M nodes $\left(y^{+}=25\right)$

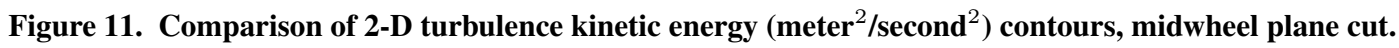




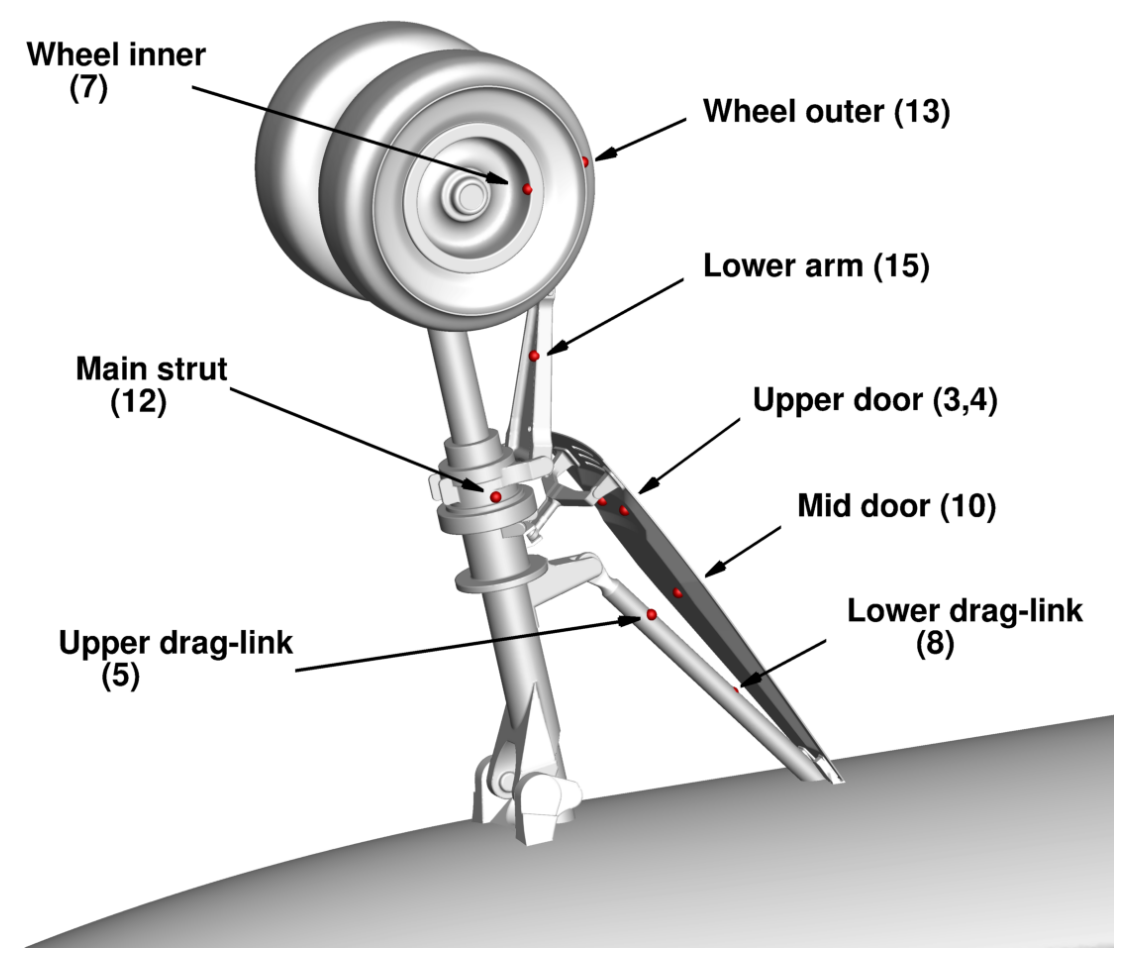

Figure 12. Surface mounted Kulite ${ }^{\circledR}$ sensor locations.
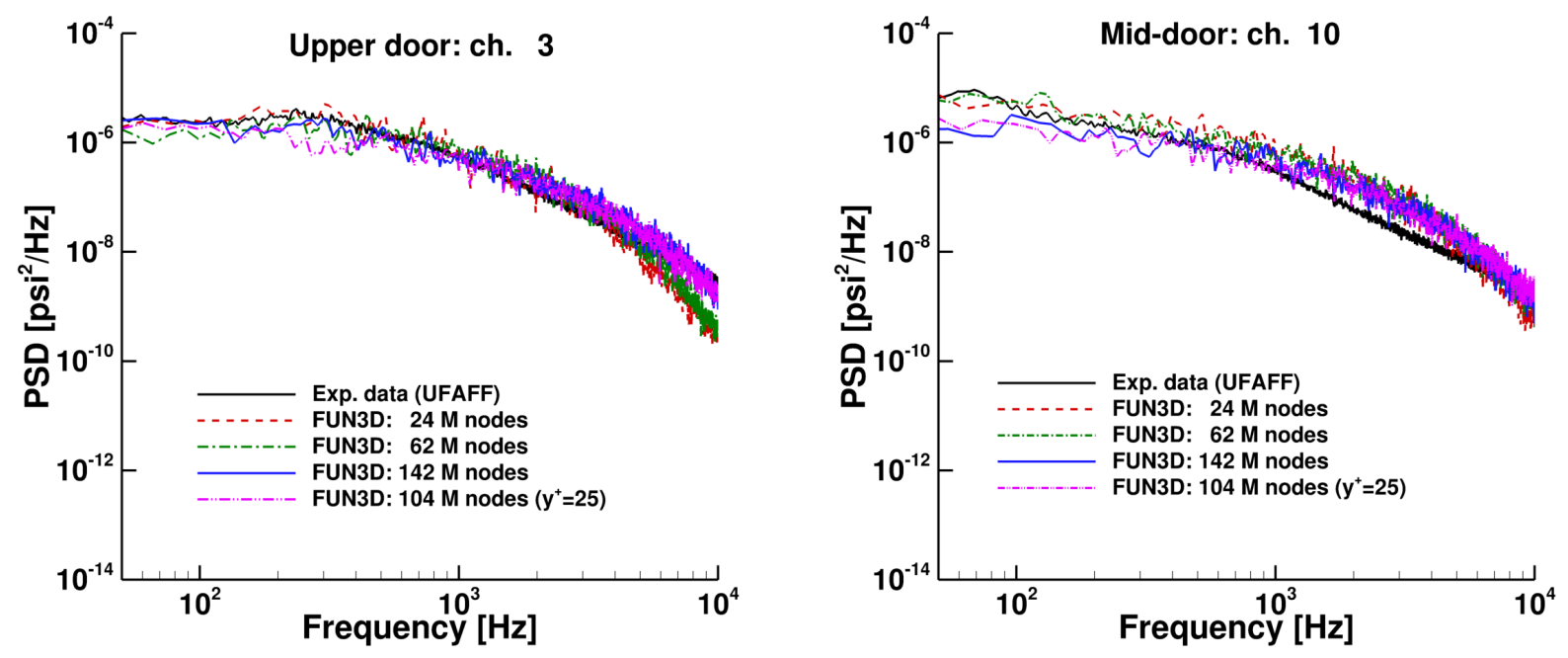

Figure 13. PSD comparisons for Kulite ${ }^{\circledR}$ sensors 3 and 10. 

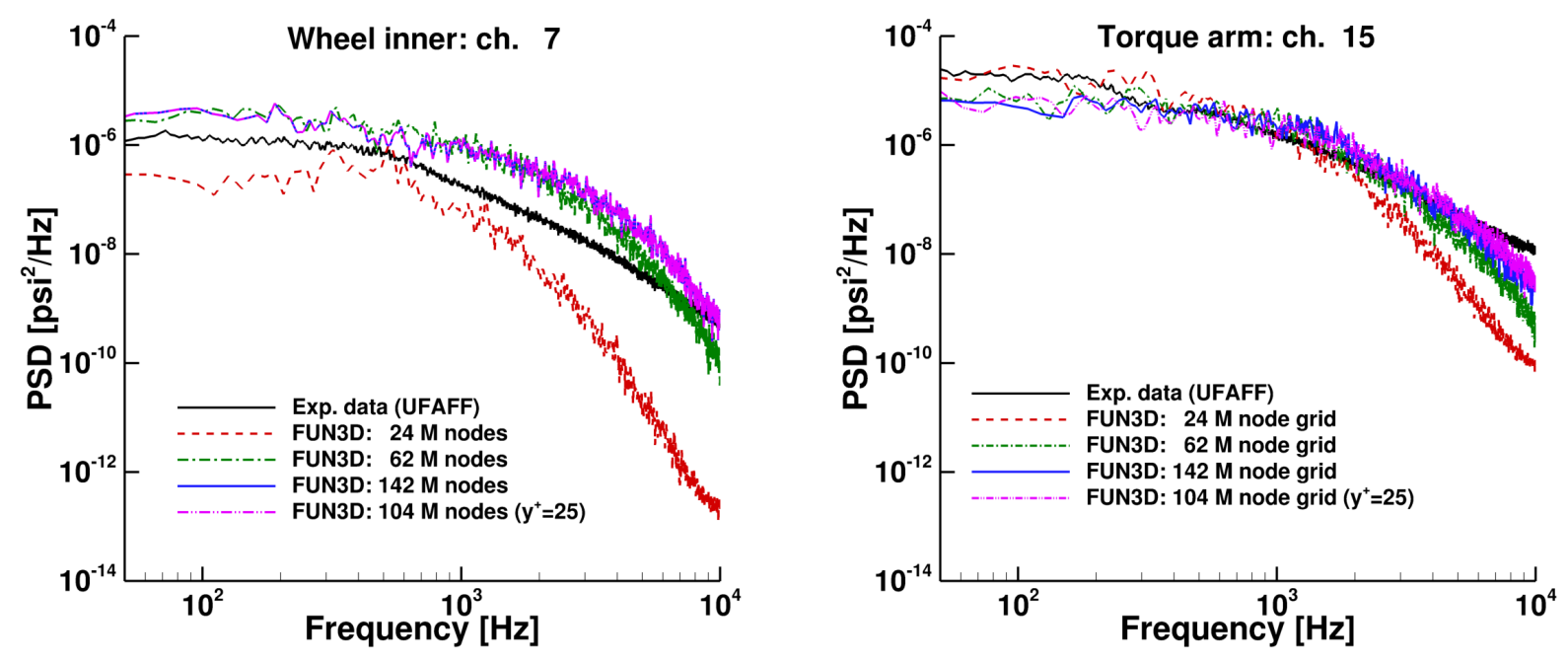

Figure 14. PSD comparisons for Kulite ${ }^{\circledR}$ sensors 7 and 15.
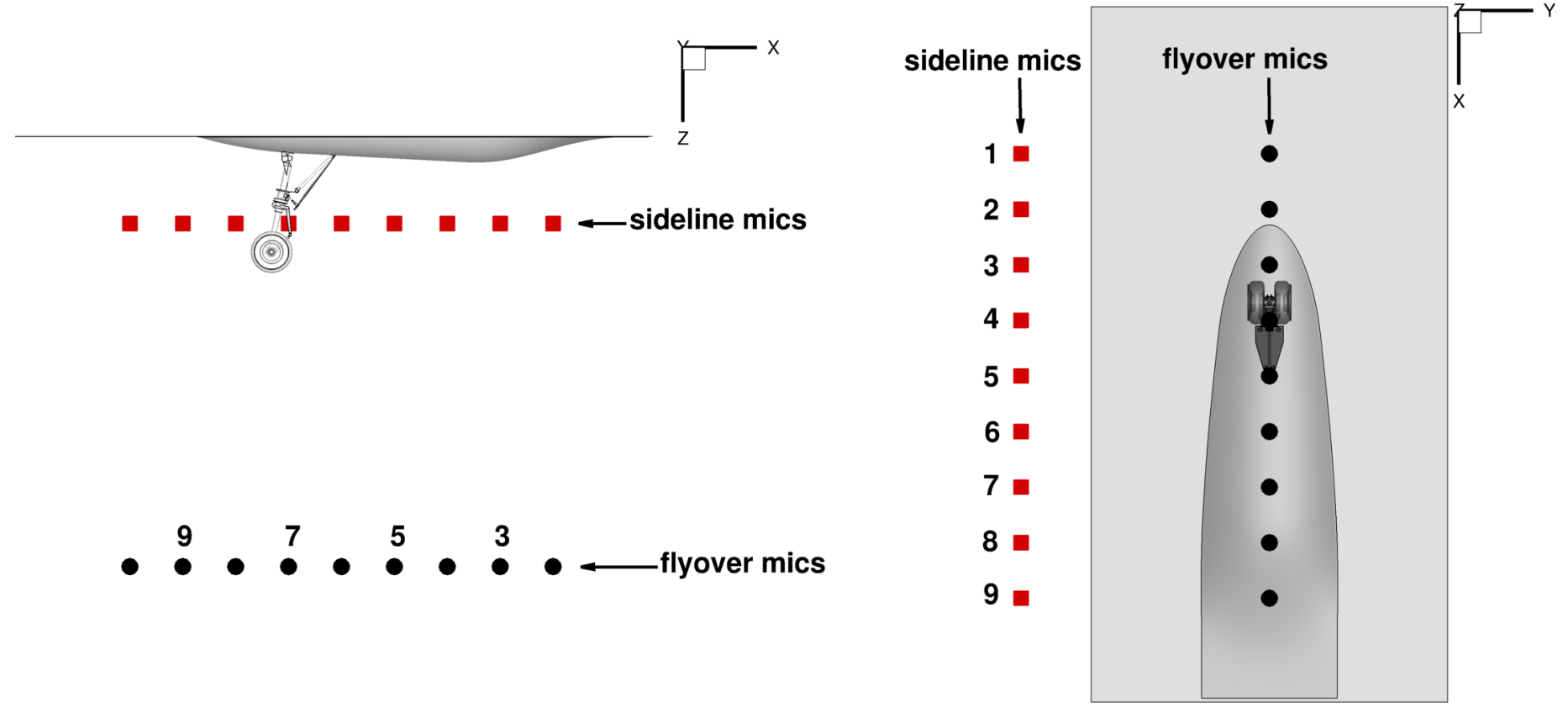

(a) side view

(b) top view

Figure 15. Linear microphone array arrangement in UFAFF. 


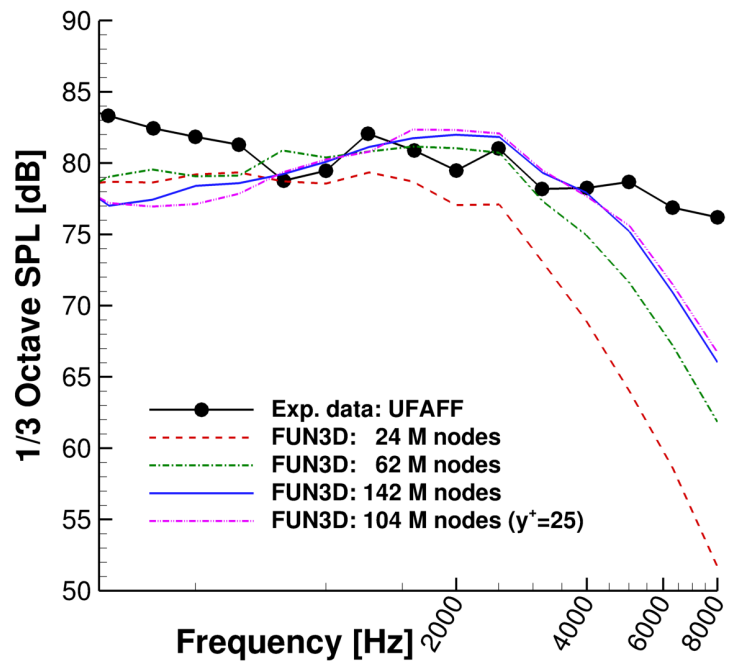

(a) Microphone 4

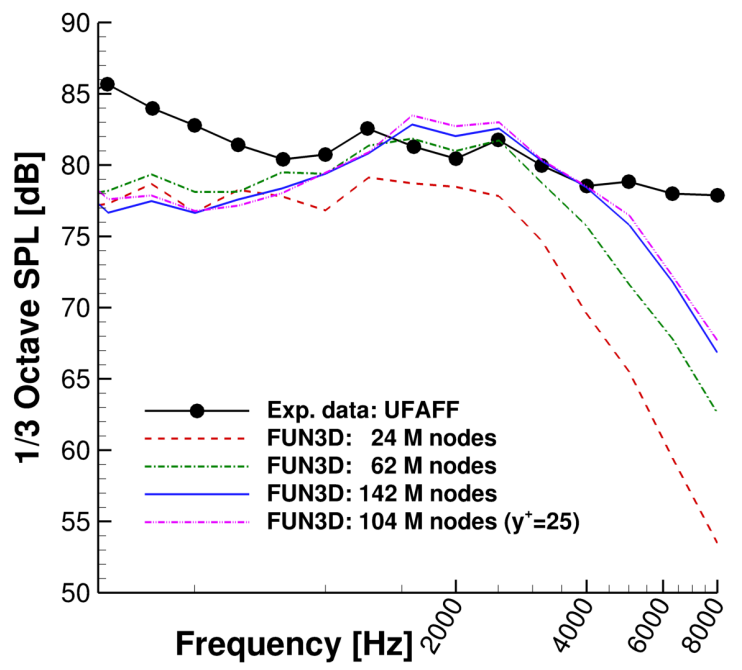

(b) Microphone 7

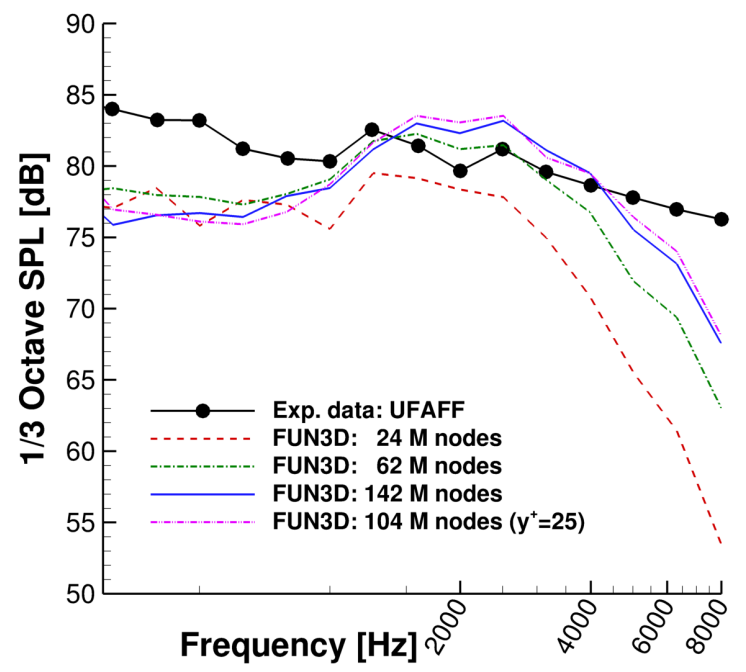

(c) Microphone 9

Figure 16. Sound pressure level comparisons at flyover microphones using solid surface perturbations in the FW-H Solver. 


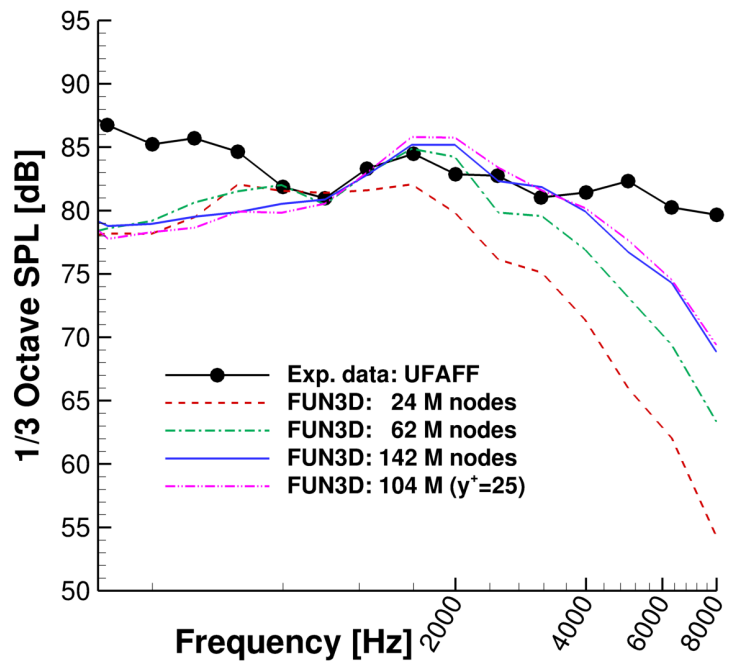

(a) Microphone 3

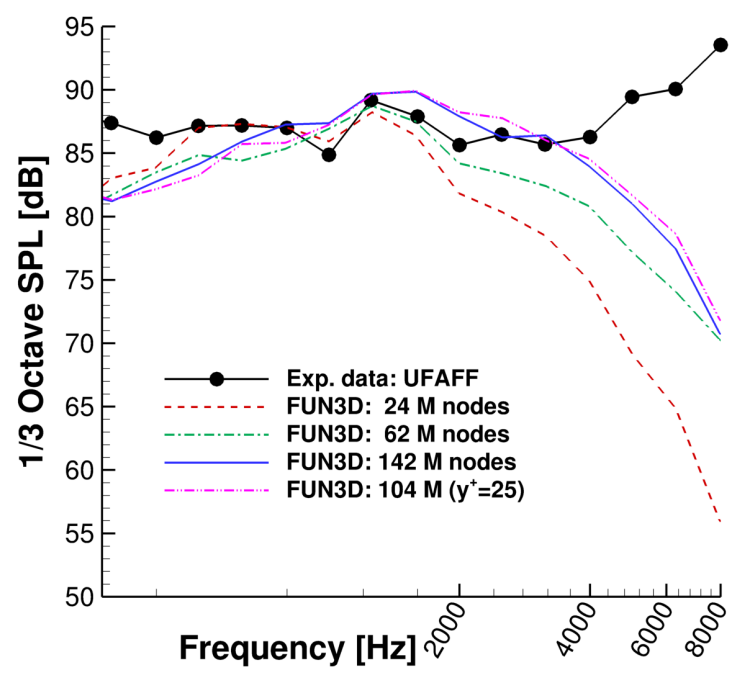

(c) Microphone 7

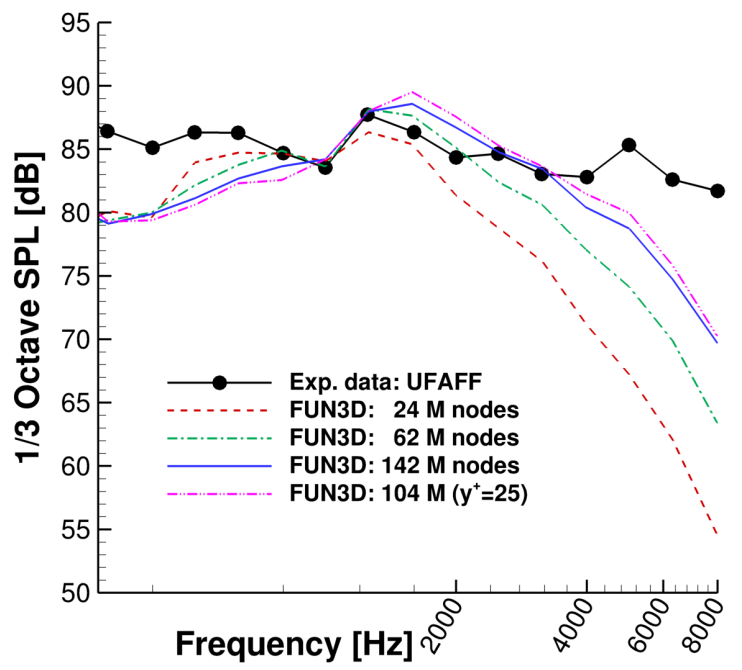

(b) Microphone 5

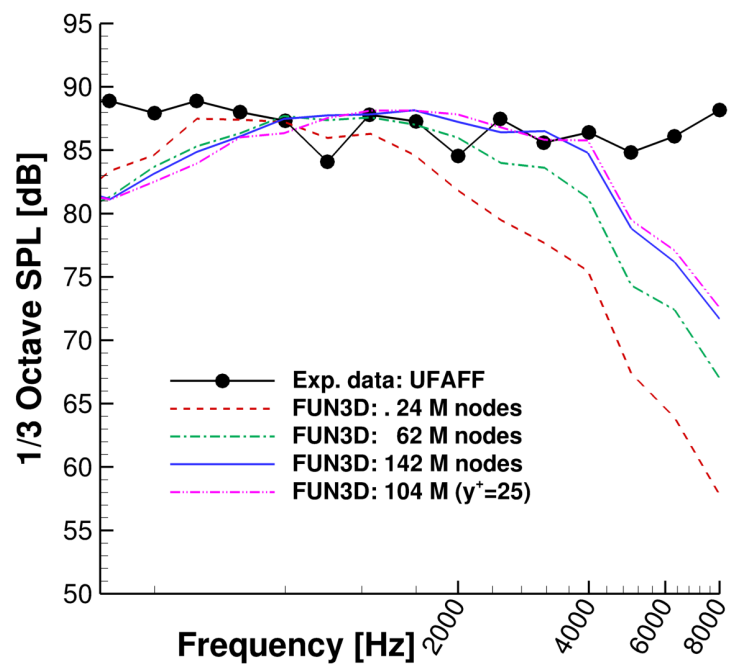

(d) Microphone 9

Figure 17. Sound pressure level comparisons at sideline microphones using solid surface perturbations in the FW-H Solver. 


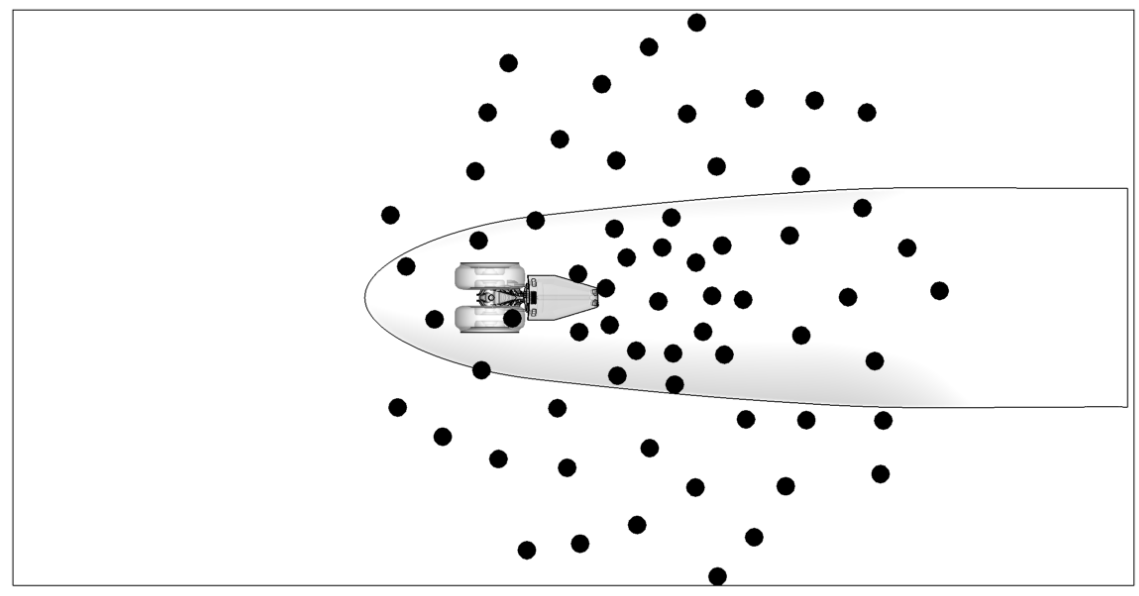

Figure 18. Linear flyover acoustic array arrangement in UFAFF.

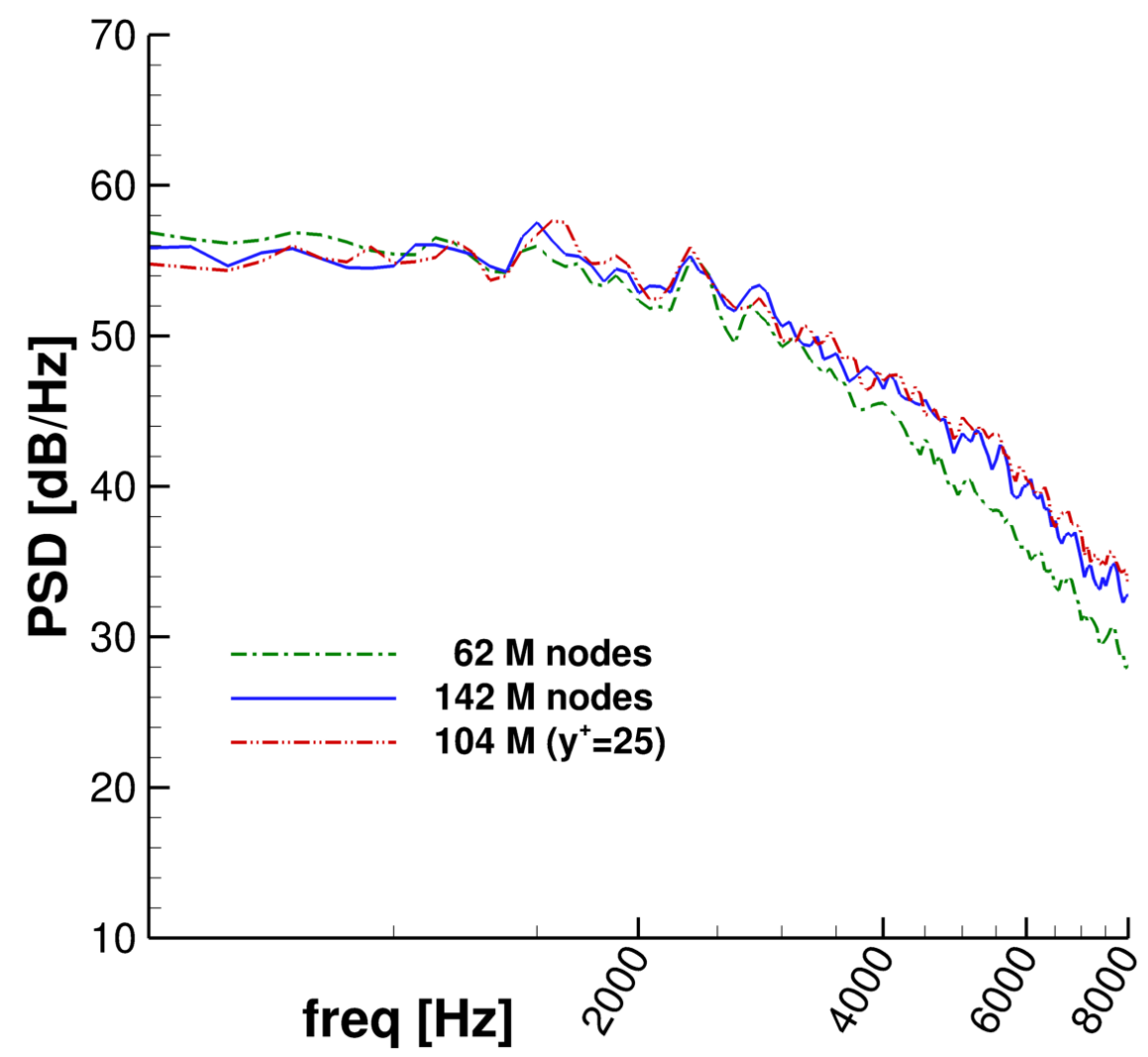

Figure 19. Noise level at the flyover acoustic array center. 

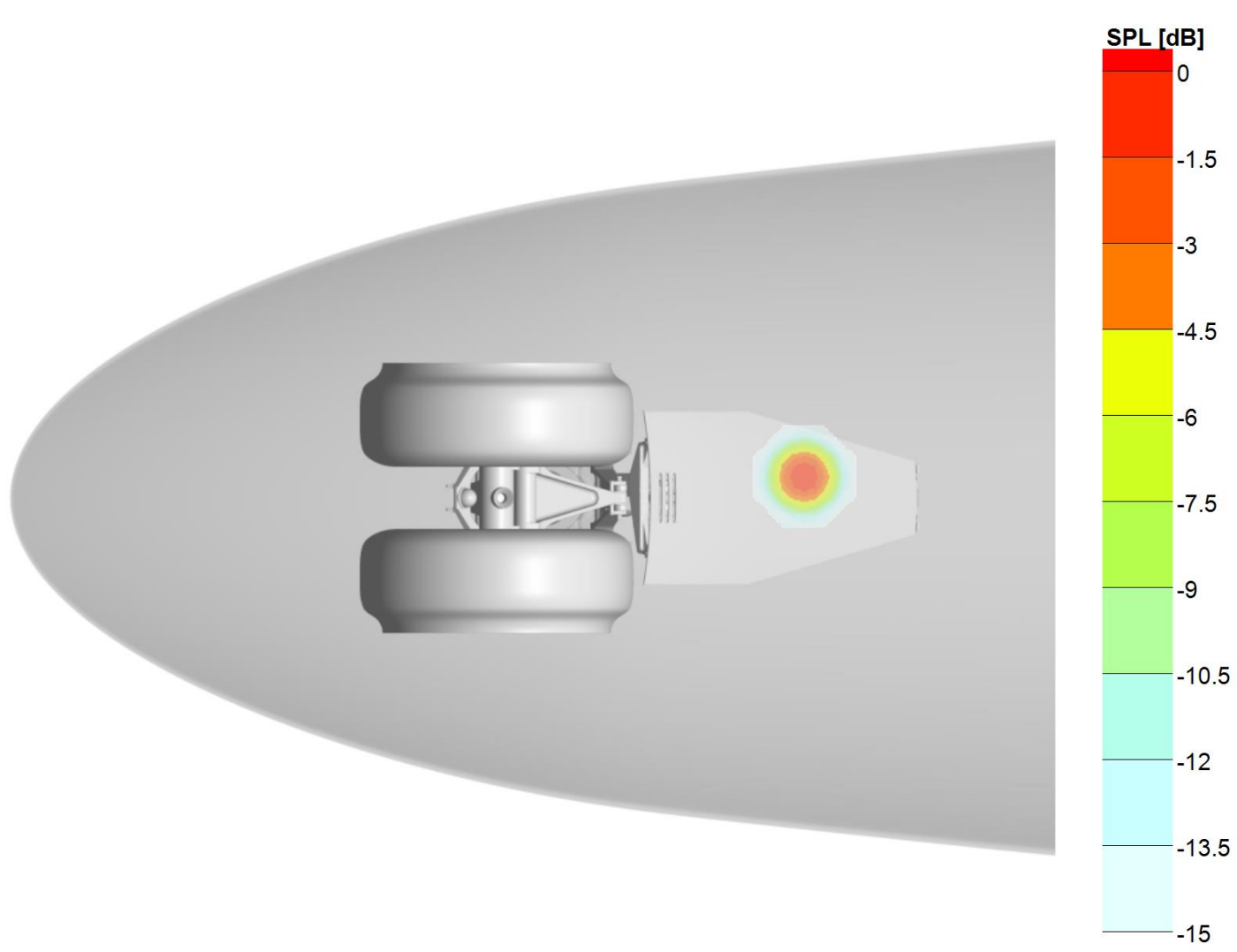

(a) $62 \mathrm{M}$ nodes

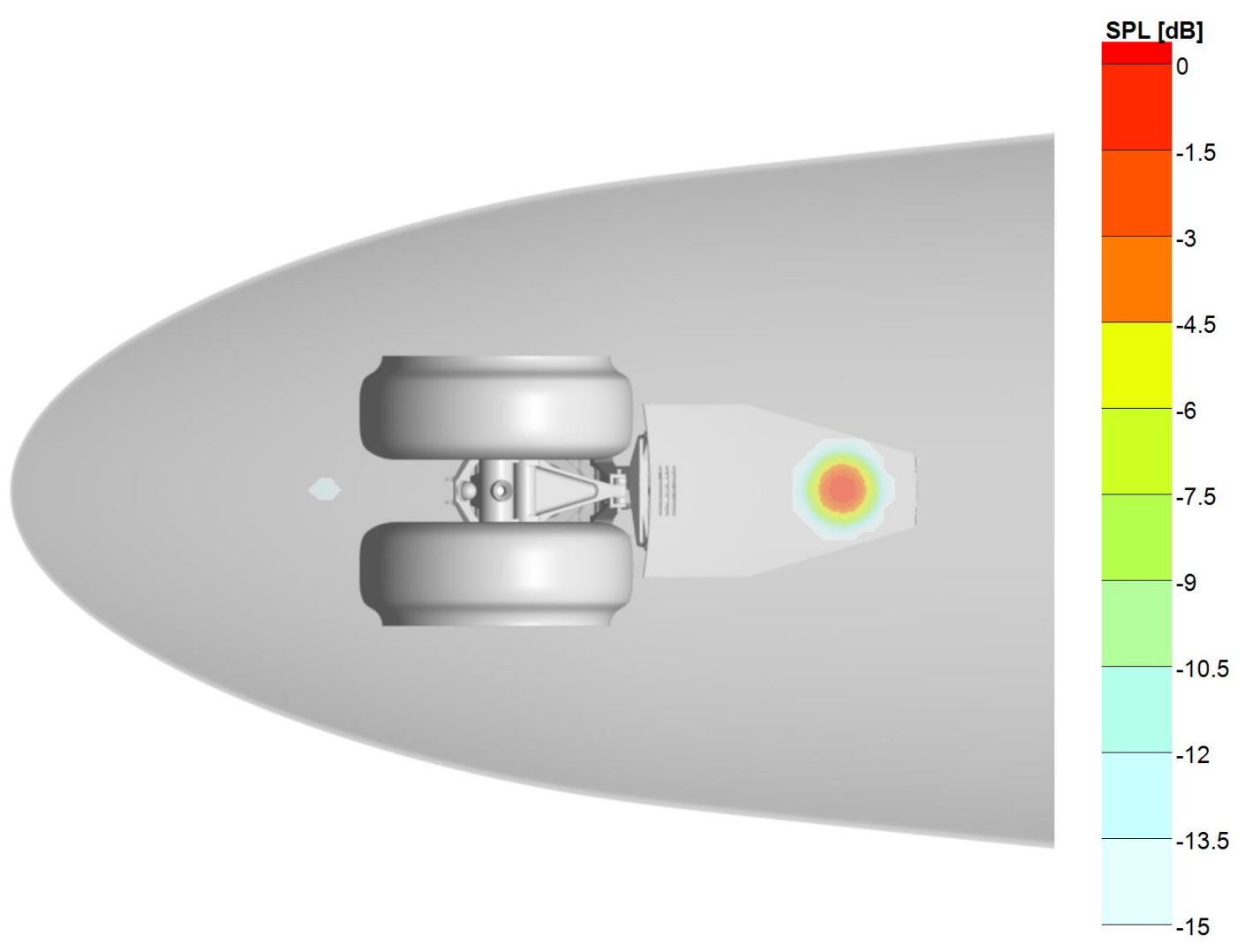

(b) $142 \mathrm{M}$ nodes

Figure 20. Comparison of flyover noise contour maps at $1 \mathrm{kHz}$. 

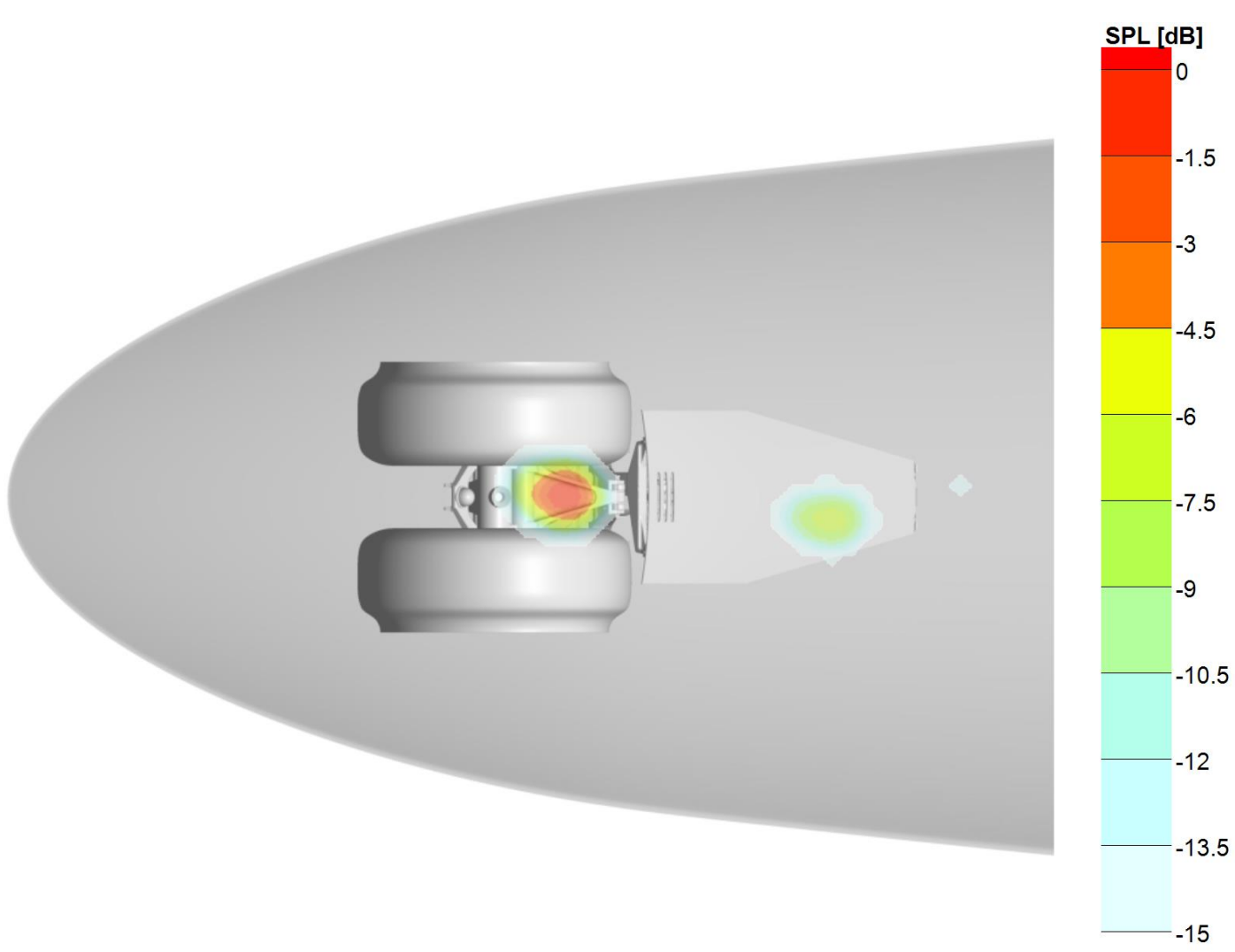

(a) $62 \mathrm{M}$ nodes

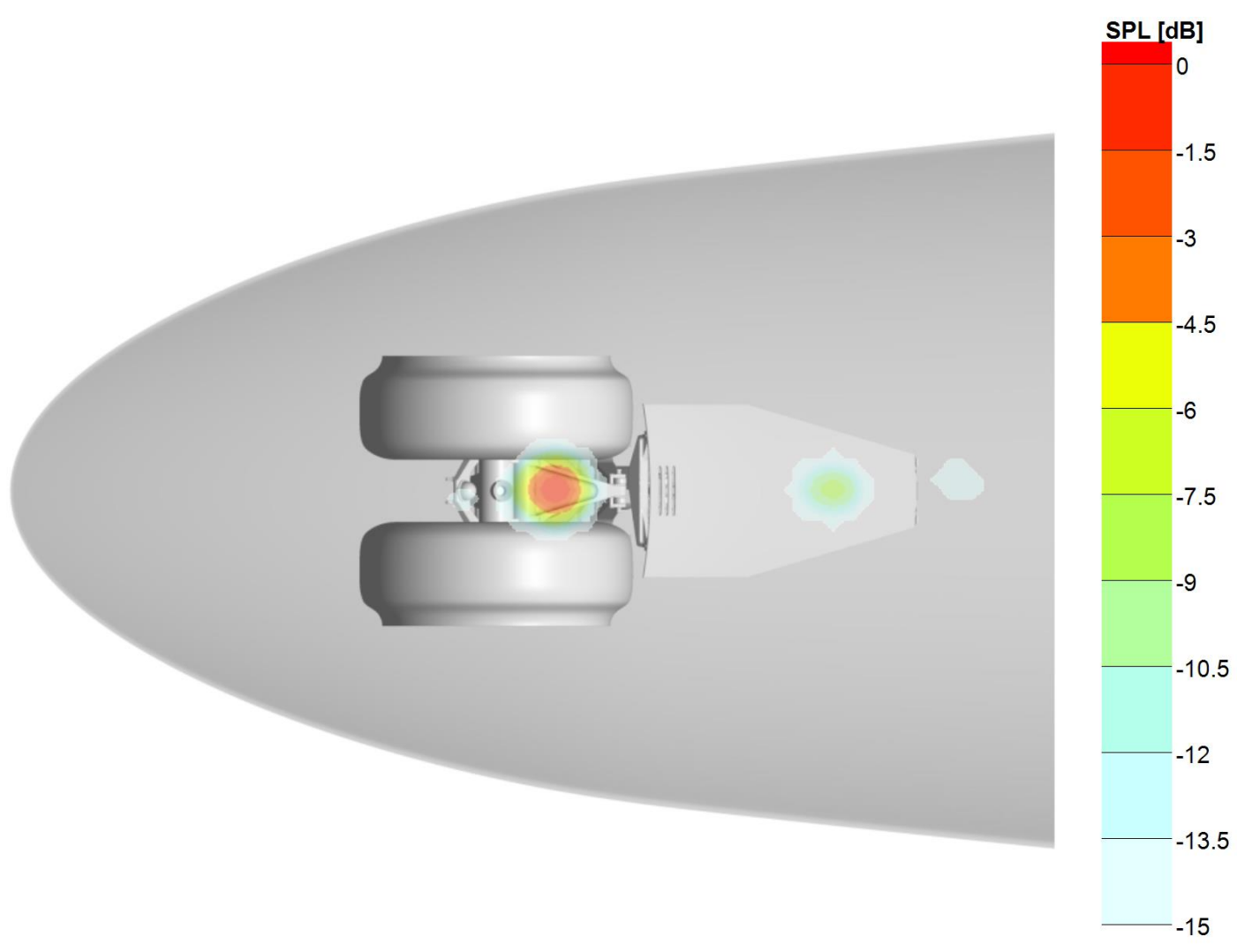

(b) $142 \mathrm{M}$ nodes

Figure 21. Comparison of flyover noise contour maps at $3 \mathrm{kHz}$. 

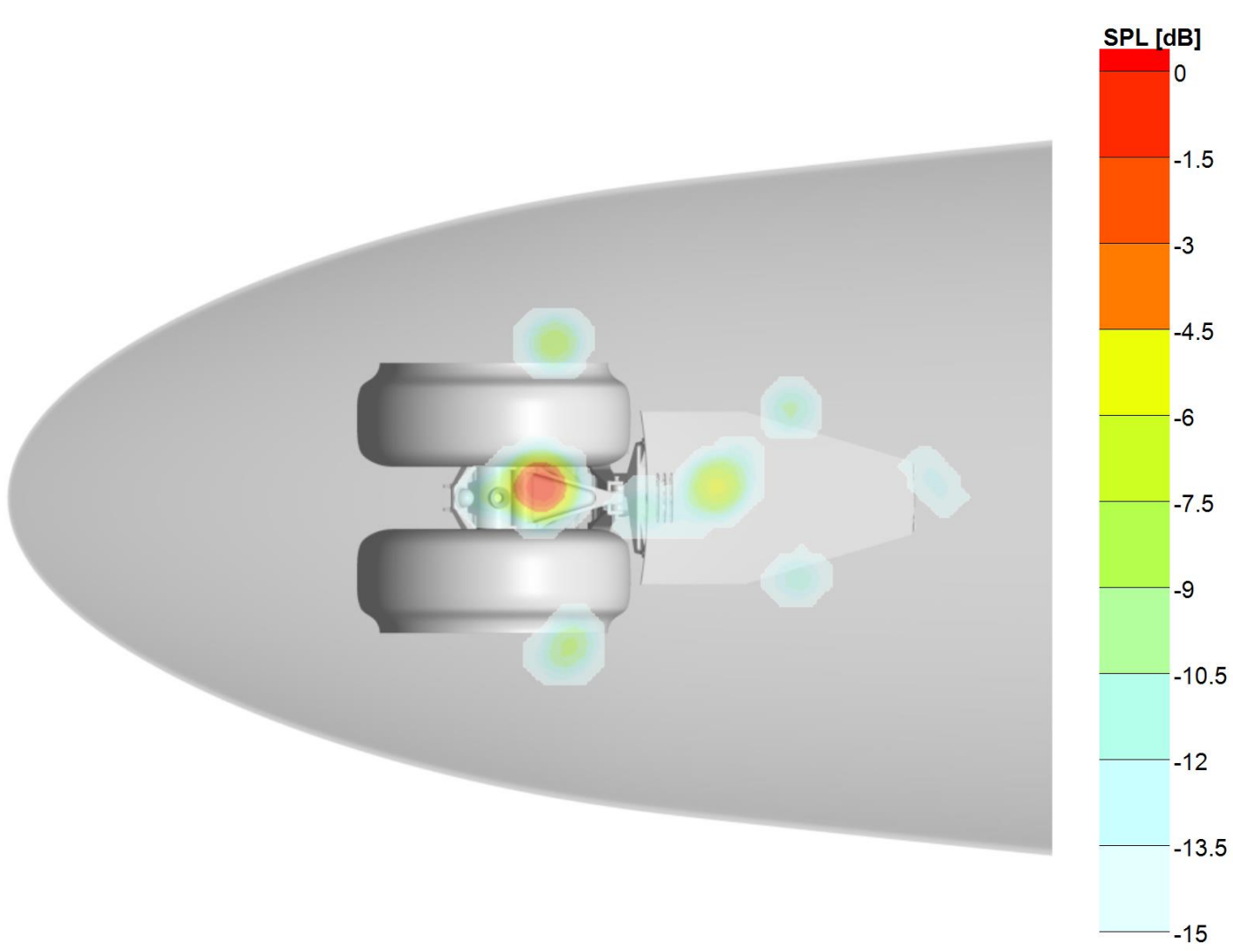

(a) $62 \mathrm{M}$ nodes

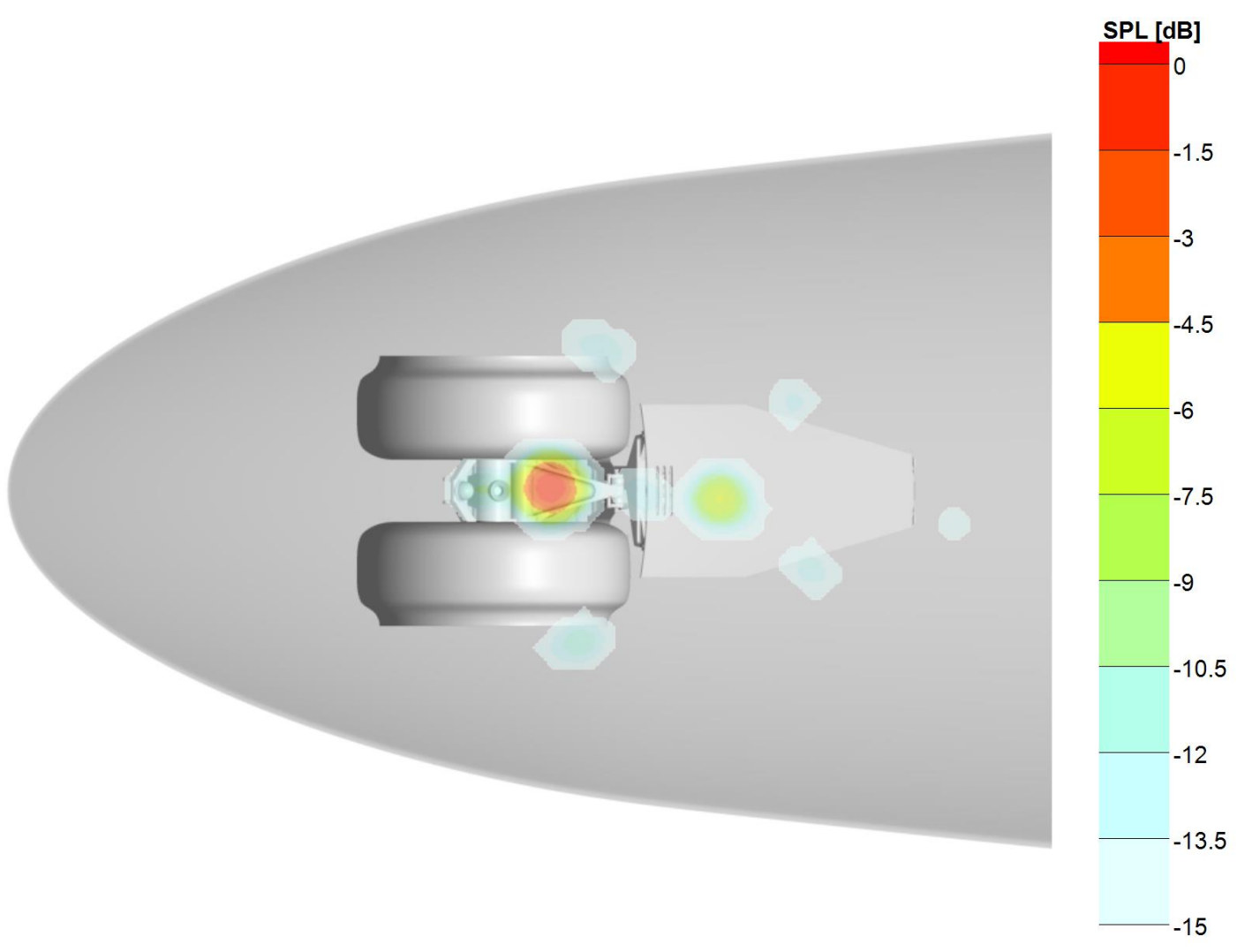

(b) $142 \mathrm{M}$ nodes

Figure 22. Comparison of flyover noise contour maps at $5 \mathrm{kHz}$. 